\title{
A Recombinant Fragment of Human Surfactant Protein D Binds Spike Protein and Inhibits Infectivity and Replication of SARS-CoV-2 in Clinical Samples
}

\author{
2 Taruna Madan ${ }^{1 *}$, Barnali Biswas ${ }^{1}$, Praveen M. Varghese ${ }^{2,3}$, Rambhadur Subedi ${ }^{1}$, Hrishikesh Pandit ${ }^{1 \ddagger}$, \\ Susan Idicula-Thomas ${ }^{4}$, Indra Kundu ${ }^{4}$, Sheetalnath Rooge ${ }^{5}$, Reshu Agarwal ${ }^{5}$, Dinesh M. Tripathi ${ }^{6}$, Savneet Kaur ${ }^{6}$, \\ Ekta Gupta $^{5}$, Sanjeev K. Gupta ${ }^{7}$, and Uday Kishore ${ }^{2^{*}}$ \\ ${ }^{1}$ Department of Innate Immunity, and ${ }^{4}$ Biomedical Informatics Centre, ICMR-National Institute for Research in Reproductive Health, \\ Mumbai, India; ${ }^{2}$ Biosciences, College of Health, Medicine and Life Sciences, Brunel University London, Uxbridge, United Kingdom; \\ ${ }^{3}$ School of Biosciences and Technology, Vellore Institute of Technology, Vellore, India; ${ }^{5}$ Department of Molecular and Cellular \\ Medicine, Institute of Liver and Biliary Sciences, Delhi, India; ${ }^{6}$ Department of Virology, Institute of Liver and Biliary Sciences, Delhi, \\ India; and ${ }^{7}$ Intrust Consulting, Mumbai, India
}

ORCID IDs: 0000-0003-2826-2443 (T.M.); 0000-0001-9502-378X (P.M.V.).

\begin{abstract}
Coronavirus disease (COVID-19) is an acute infectious disease caused by the severe acute respiratory syndrome coronavirus 2 (SARS-CoV-2). Human SP-D (surfactant protein D) is known to interact with the spike protein of SARS-CoV, but its immune surveillance against SARS-CoV-2 is not known. The current study aimed to examine the potential of a recombinant fragment of human SP-D (rfhSP-D) as an inhibitor of replication and infection of SARS-CoV-2. The interaction of $\mathrm{rfhSP}-\mathrm{D}$ with the spike protein of SARS-CoV-2 and human ACE-2 (angiotensin-converting enzyme 2) receptor was predicted via docking analysis. The inhibition of interaction between the spike protein and ACE-2 by rfhSP-D was confirmed using direct and indirect ELISA. The effect of rfhSP-D on replication and infectivity of SARS-CoV-2 from clinical samples was assessed by measuring the expression of $R d R p$ gene of the virus using quantitative PCR. In silico interaction
\end{abstract}

studies indicated that three amino acid residues in the receptorbinding domain of spike protein of SARS-CoV-2 were commonly involved in interacting with rfhSP-D and ACE-2. Studies using clinical samples of SARS-CoV-2-positive cases (asymptomatic, $n$ $=7$; symptomatic, $n=8)$ and negative control samples $(n=15)$ demonstrated that treatment with $1.67 \mu \mathrm{M}$ rfhSP-D inhibited viral replication by $\sim 5.5$-fold and was more efficient than remdesivir $(100 \mu \mathrm{M})$ in Vero cells. An approximately two-fold reduction in viral infectivity was also observed after treatment with $1.67 \mu \mathrm{M}$ rfhSP-D. These results conclusively demonstrate that the rfhSP-D mediated calcium independent interaction between the receptorbinding domain of the S1 subunit of the SARS-CoV-2 spike protein and human ACE-2, its host cell receptor, and significantly reduced SARS-CoV-2 infection and replication in vitro.

Keywords: SARS-CoV-2; COVID-19; surfactant protein D; spike protein; entry inhibitor

(Received in original form January 4, 2021; accepted in final form March 30, 2021)

a This article is open access and distributed under the terms of the Creative Commons Attribution Non-Commercial No Derivatives License 4.0 (https://creativecommons.org/licenses/by-nc-nd/4.0/). For commercial usage and reprints, please contact Diane Gern (dgern@thoracic.org).

${ }^{*}$ Co-senior authors.

FPresent address: National Cancer Institute at Frederick, Frederick, Maryland

Supported by an intramural grant from ICMR-NIRRH (accession number RA/1002/12-2020).

Author Contributions: T.M., B.B., P.M.V., and R.S. performed protein-related work. S.I.-T. and I.K. performed molecular modeling and mutational studies. T.M., S.R., R.A., D.M.T., S.K., E.G., and S.K.G. contributed to the clinical samples and infection assay. T.M., P.M.V., H.P., S.K.G., and U.K. analyzed and interpreted the data and prepared the manuscript. T.M., S.K.G., and U.K. performed conception and design. T.M., P.M.V., E.G., S.K.G., and U.K. performed data analysis. T.M. and U.K. performed interpretation. T.M., B.B., P.M.V., and U.K. drafted the first version of the manuscript. T.M., H.P., and U.K. reviewed and edited the manuscript.

Correspondence and requests for reprints should be addressed to Uday Kishore, Ph.D., Biosciences, College of Health, Medicine and Life Sciences, 241, Heinz Wolff Building, Brunel University London, Uxbridge UB8 3PH, UK. E-mail: uday.kishore@brunel.ac.uk.

Author disclosures are available with the text of this article at www.atsjournals.org.

This article has a data supplement, which is accessible from this issue's table of contents at www.atsjournals.org.

Am J Respir Cell Mol Biol Vol 65, Iss 1, pp 41-53, July 2021

Originally Published in Press as DOI:10.1165/rcmb.2021-0005OC on March 30, 2021

Copyright (@) 2021 by the American Thoracic Society

Internet address: www.atsjournals.org 
The coronavirus disease (COVID-19) pandemic, caused by the severe acute respiratory syndrome coronavirus-2 (SARSCoV-2) $(1,2)$, has affected $\sim 58$ million people across the globe and has claimed more than 1 million lives within its first year (3). The SARS-CoV-2 spike protein (S protein) is cleaved into S1 subunit, which is involved in host receptor binding, and S2 subunit, which is involved in membrane fusion, by the host's TMPRSS2

(transmembrane serine protease 2) (4). This priming of the $\mathrm{S}$ protein by host proteases enables it to bind with the ACE2

(angiotensin-converting enzyme 2) receptor on the nasopharyngeal epithelial cells, leading to its entry into the host cell (4). Although vaccines against the virus are being continually developed and trialed, therapeutic strategies to treat severe COVID19 patients are limited and comprise remdesivir and dexamethasone (5).

The innate immune system plays a crucial role against SARS-CoV-2 infection; the majority of infected individuals purge the virus within a few days with minimal involvement of adaptive immune response (6). Collectins are a group of humoral pattern recognition receptors, of which human SP-D (pulmonary surfactant protein D) is known to act as a potent viral entry inhibitor, including against human immunodeficiency virus 1 and influenza A virus $(7,8)$. The primary structure of SP-D is characterized by an N-terminus that is involved in multimerization, a triple-helical collagenous region made up of Gly-X-Y repeats, an $\alpha$-helical coiled-coil neck region, and a C-terminal C-type lectin or carbohydrate recognition domain (CRD) (9). The protective effects of SP-D against a range of bacterial, viral, and fungal pathogens, leading to their agglutination, growth inhibition, enhanced phagocytosis, neutralization, and modulation of immune responses, are well documented $(9,10)$.

During the SARS-CoV epidemic in 2002, elevated concentrations of SP-D were reported in the serum of the patients infected with highly pathogenic $\beta-\mathrm{CoV}$ SARS-CoV (11). Purified SP-D has been shown to bind to the receptor-binding domain (RBD) of the glycosylated S protein of SARS-CoV, which shares $74 \%$ homology with the RBD of SARS-CoV-2 (12). In addition, SP-D also binds $\alpha-\mathrm{CoV}$ and $\mathrm{HCoV}-229 \mathrm{E}$ and inhibits infection in human bronchial epithelial cells (13). These mounting pieces of evidence encouraged exploration of the therapeutic potential of SP-D in patients with COVID-19.

In this study, we used a wellcharacterized recombinant fragment of human SP-D (rfhSP-D) comprising homotrimeric neck and CRD region to study its protective effect against SARS-CoV-2 infection. As the recombinant fragment has the advantages of a smaller size to reach the distal lung locations and higher resistance to proteases and collagenases over the fulllength SP-D, we evaluated the interaction of rfhSP-D with RBD and the spike of SARS$\mathrm{CoV}-2$, and its inhibitory potential against infection and replication of SARS-CoV-2 in clinical samples.

Some of the results from this study have been previously reported in the form of a preprint (bioRxiv, [18 December 2020] https://doi.org/10.1101/2020.12.18.423415).

\section{Methods}

An additional detailed section for performing these experiments is provided as a supplement.

\section{Clinical Samples}

The clinical samples (throat and nasal swabs) $(n=15)$ (Table 1$)$ were obtained from symptomatic contacts of lab-confirmed cases (category 2) $(n=2)$, hospitalized patients with severe acute respiratory infections (category 4$)(n=3)$, asymptomatic direct and high-risk contacts of lab-confirmed cases (category 5a) $(n=7)$, and hospitalized patients with symptomatic influenza-like illness (category 6) $(n=3)$, who had tested positive by RT-PCR for SARS-CoV-2. Samples $(n=15)$ that tested negative by RT-PCR for SARS-CoV-2 were used as control. The inclusion criteria for the cases were age (between 18 and $45 \mathrm{yr}$ ) and a cycle threshold value (Ct value) between 10 and 15 (18-45 yr and an undetectable viral load for control samples). The two groups showed no significant difference in their age and sex distribution. The 50\% tissue culture infective dose $\left(\mathrm{TCID}_{50}\right)$ of the clinical samples was estimated using a template that used the Spearman and Kärber method for calculation (14). The estimated $\mathrm{TCID}_{50}$ value was further confirmed via MTT assay. Briefly, $5 \times 10^{4}$ Vero cells in Vero growth media (minimal essential medium [MEM] Glutamax $+10 \%$ FBS, $1 \%$ penicillinstreptomycin, and $1 \%$ sodium pyruvate;
Gibco) were grown in a 96-well plate overnight. The clinical samples from the cases and controls at various dilutions were added to the cells and incubated for 1 hour, wells were washed with PBS twice, and fresh Vero growth medium was added to the cells and incubated $\left(96 \mathrm{~h} ; 37^{\circ} \mathrm{C} ; 5 \% \mathrm{CO}_{2}\right)$. An MTT assay was performed to assess cell viability.

\section{In Silico Analysis of rfhSP-D Interaction with SARS-CoV-2 S Protein and Human ACE-2}

Structural information with respect to the molecular interactions between $S$ protein and human ACE-2 (hACE-2) is available $(15,16)$. The rfhSP-D trimer (Protein Data Bank [pdb] identification 1PW9) was blinddocked with 1) RBD of S protein in open conformation (pdb identification 6VYB), and 2) dimeric hACE-2 (pdb identification 6VW1). Top 100 docked poses selected were further refined using the FireDock web server $(17,18)$ for calculating global free energy. The top five refined structures were filtered on the basis of interactions between the receptor-binding motif (RBM) of $S$ protein, C-type lectin domain (aa 240-355) of rfhSP-D, and N-terminal of hACE-2.

The effect of binding of trimeric rfhSP$\mathrm{D}$ to $\mathrm{S}$ protein and dimeric ACE-2 on ACE-2-S protein interaction was evaluated by further docking the complex of 1) $\mathrm{S}$ protein and rfhSP-D with hACE-2 and 2) hACE-2 and rfhSP-D with S protein. Patchdock web server $(19,20)$ was used for all docking experiments.

\section{In Silico Interaction of Single-Residue rfhSP-D Mutants with S Protein and hACE-2}

The importance of rfhSP-D residues predicted to interact with ACE-2 and S protein was further validated using singleresidue mutations in the docked complex using the mCSM-PPI2 web server (21). Each of the rfhSP-D residues involved in interaction with virus-binding hotspot of ACE-2 and RBD of S protein were mutated to the standard amino acids, and its effect on the binding energy of the complex was assessed.

\section{ELISA}

The rfhSP-D used was expressed and purified from Escherichia coli, as described previously $(22,23)$. rfhSP-D binding to $S$ protein or $\mathrm{RBD}$ was analyzed using the SARS-CoV-2 Inhibitor Screening Kit (EP-105; ACRO Biosystems). 
Table 1. Characteristics of Clinical Samples Used in the Study

\begin{tabular}{|c|c|c|c|c|c|}
\hline Sample & Age (yr) & Sex & Ct Value for SARS-CoV-2 E Gene & Ct Value for SARS-CoV-2 RdRp Gene & Category \\
\hline $1 S$ & 32 & M & 18.5 & 15.46 & 2 \\
\hline $2 S$ & 41 & $\mathrm{~F}$ & 17.48 & 15.36 & 6 \\
\hline $3 S$ & 44 & M & 13.46 & 12.01 & 6 \\
\hline $4 S$ & 39 & $\mathrm{~F}$ & 13.22 & 12.67 & 2 \\
\hline $5 S$ & 36 & M & 12.08 & 14.55 & 6 \\
\hline $6 S$ & 42 & $\mathrm{~F}$ & 12.28 & 9.4 & 4 \\
\hline $7 S$ & 37 & $\mathrm{M}$ & 16.26 & 15.42 & 4 \\
\hline $8 S$ & 28 & $\mathrm{~F}$ & 17.15 & 15.62 & 4 \\
\hline 9AS & 32 & M & 10.45 & 10.8 & $5 a$ \\
\hline $10 A S$ & 31 & $\mathrm{~F}$ & 12.32 & 12.4 & $5 a$ \\
\hline 11AS & 26 & $\mathrm{M}$ & 15.33 & 12.52 & $5 a$ \\
\hline $12 A S$ & 35 & $\mathrm{~F}$ & 10.59 & 12.4 & $5 a$ \\
\hline 13AS & 38 & $\mathrm{M}$ & 15.77 & 12.89 & $5 a$ \\
\hline $14 \mathrm{AS}$ & 29 & $\mathrm{~F}$ & 18.07 & 10.72 & $5 a$ \\
\hline $15 A S$ & 33 & $\mathrm{M}$ & 15.64 & 13.81 & $5 a$ \\
\hline $1 \mathrm{C}$ & 33 & M & $\mathrm{Nd}$ & $\mathrm{Nd}$ & Control \\
\hline $2 \mathrm{C}$ & 39 & $\mathrm{~F}$ & $\mathrm{Nd}$ & $\mathrm{Nd}$ & Control \\
\hline $3 C$ & 45 & $M$ & $\mathrm{Nd}$ & $\mathrm{Nd}$ & Control \\
\hline $4 C$ & 39 & $\mathrm{~F}$ & $\mathrm{Nd}$ & $\mathrm{Nd}$ & Control \\
\hline $5 C$ & 34 & $M$ & $\mathrm{Nd}$ & $\mathrm{Nd}$ & Control \\
\hline $6 C$ & 44 & $\mathrm{~F}$ & $\mathrm{Nd}$ & $\mathrm{Nd}$ & Control \\
\hline 7C & 35 & M & $\mathrm{Nd}$ & $\mathrm{Nd}$ & Control \\
\hline $8 \mathrm{C}$ & 30 & $\mathrm{~F}$ & $\mathrm{Nd}$ & $\mathrm{Nd}$ & Control \\
\hline $9 \mathrm{C}$ & 30 & $M$ & $\mathrm{Nd}$ & $\mathrm{Nd}$ & Control \\
\hline $10 \mathrm{C}$ & 32 & $\mathrm{~F}$ & $\mathrm{Nd}$ & $\mathrm{Nd}$ & Control \\
\hline $11 \mathrm{C}$ & 29 & M & $\mathrm{Nd}$ & $\mathrm{Nd}$ & Control \\
\hline $12 \mathrm{C}$ & 33 & $\mathrm{~F}$ & $\mathrm{Nd}$ & $\mathrm{Nd}$ & Control \\
\hline $13 \mathrm{C}$ & 40 & $M$ & $\mathrm{Nd}$ & $\mathrm{Nd}$ & Control \\
\hline${ }^{14} \mathrm{C}$ & 32 & $\mathrm{~F}$ & $\mathrm{Nd}$ & $\mathrm{Nd}$ & Control \\
\hline $15 C$ & 30 & $M$ & $\mathrm{Nd}$ & $\mathrm{Nd}$ & Control \\
\hline
\end{tabular}

Definition of abbreviations: $\mathrm{Ct}=$ cycle threshold; $\mathrm{Nd}=$ not detected; RdRp $=\mathrm{RNA}$-dependent RNA polymerase; SARS-CoV-2 $=$ severe acute respiratory syndrome coronavirus 2 .

Microtiter wells were coated with 0.3 $\mu \mathrm{g} / \mathrm{ml}(0.54 \mathrm{nM}) \mathrm{S}$ protein and incubated with rfhSP-D $(20,10$, and $5 \mu \mathrm{g} / \mathrm{ml}$ or 0.334 , 0.167 , and $0.083 \mu \mathrm{M})$, or full-length (FL) SP$\mathrm{D}(20 \mu \mathrm{g} / \mathrm{ml}$ or $0.038 \mu \mathrm{M})$ for 1 hour at $37^{\circ} \mathrm{C}$ in PBS supplemented with $5 \mathrm{mM}$ $\mathrm{CaCl}_{2}$. After blocking and washing, the wells were incubated with polyclonal or monoclonal antibodies against SP-D (1 mg/ $\mathrm{ml})$, respectively, and probed with their respective secondary antibodies conjugated with horseradish peroxidase (HRP). The binding was detected using $3,3^{\prime}, 5,5^{\prime}$ tetramethylbenzidine substrate, and absorbance was recorded at $450 \mathrm{~nm}$. Similarly, to ascertain the calcium dependence of the interaction, a binding assay was performed with $10 \mathrm{mM}$ EDTA.

rfhSP-D/FL SP-D and ACE-2 interaction was evaluated by coating rfhSP-D $(0.1 \mu \mathrm{g} / \mathrm{ml}$ or $1.67 \mathrm{nM}) / \mathrm{FL}$ SP-D $(0.1 \mu \mathrm{g} / \mathrm{ml}$ or $0.19 \mathrm{nM})$ and probing with biotinylated hACE- $2(0.12,0.06$, and $0.00 \mu \mathrm{g} / \mathrm{ml}$ or 0.52 , $0.26,0.0 \mathrm{nM})$, followed by the addition of streptavidin tagged with HRP.
To assess whether rfhSP-D inhibited the interaction between $S$ protein/RBD and biotinylated hACE-2, rfhSP-D (5, 1 , and 0 $\mu \mathrm{g} / \mathrm{ml}$ or $83.5,16.7$, and $0.0 \mathrm{nM}$ ) was preincubated with the coated $S$ protein/RBD, followed by the addition of biotinylated ACE-2. The S protein-hACE-2 binding was probed with the HRP-tagged streptavidin antibody. rfhSP-D $(5 \mu \mathrm{g} / \mathrm{ml}$ or $83.5 \mathrm{nM})$ in $10 \mathrm{mM}$ EDTA was also used.

\section{Vero Cell Replication Assay}

Vero cells (CCL-81; American Type Culture Collection) $\left(5 \times 10^{4}\right)$ were cultured in serum-free MEM. SARS-CoV-2-positive clinical samples (100 $\mathrm{TCID}_{50} /$ well; multiplicity of infection [MOI], 0.01) were preincubated with rfhSP-D $(0 \mu \mathrm{g} / \mathrm{ml}, 50 \mu \mathrm{g} /$ $\mathrm{ml}$, and $100 \mu \mathrm{g} / \mathrm{ml}$ or $0,0.835$, and $1.67 \mu \mathrm{M}$ ) in MEM containing $5 \mathrm{mM} \mathrm{CaCl}_{2}$ for 1 hour at room temperature and 1 hour at $4^{\circ} \mathrm{C}(7)$. SARS-CoV-2-negative clinical samples (volume equivalent to $100 \mathrm{TCID}_{50}$ of the ageand sex-matched SARS-CoV-2-positive sample/well; MOI, 0.01) were used as a control. This pretreated or untreated virus was added to the cells and incubated for 1 hour at $37^{\circ} \mathrm{C}\left(5 \% \mathrm{CO}_{2}\right)$. After PBS washes, infection medium (MEM + 0.3\% BSA) was added and incubated for 24 hours to assess viral replication. Total RNA was extracted from the cell pellet using the Perkin Elmer automated extractor. Real-time qPCR for SARS-CoV-2 was performed using Pathodetect kits (MyLabs). For the replication analysis, the Ct value for SARSCoV-2 RdRp (RNA-dependent RNA polymerase) gene was used. Cells incubated with rfhSP-D without virus were used as the protein control.

\section{Vero Cell Infection Assay}

SARS-CoV-2-positive clinical samples (500 TCID $_{50}$ /well; MOI, 0.05) and SARS-CoV-2 negative clinical samples (equivalent volume) were treated with rfhSP-D (7). After adding the infection medium, Vero cells $\left(5 \times 10^{5}\right)$ were incubated for 2 hours and harvested, and real-time qPCR was performed, as described above. 
Table 2. Results of Docking of S Protein, ACE-2, and rfhSP-D

\begin{tabular}{|c|c|c|c|c|c|}
\hline \multirow[b]{2}{*}{ S. Number } & \multirow[b]{2}{*}{ Receptor } & \multirow[b]{2}{*}{ Ligand } & \multirow[b]{2}{*}{ Binding Energy (kcal/mol) } & \multicolumn{2}{|r|}{ Interactions } \\
\hline & & & & Receptor & Ligand $^{\dagger}$ \\
\hline 1 & ACE-2 & S Protein & Crystal structure & $\begin{array}{l}\text { Ser19 } \\
\text { Gln24 } \\
\text { Lys31 } \\
\text { His34 } \\
\text { Glu35 } \\
\text { Glu37 } \\
\text { Asp38 } \\
\text { Tyr41 } \\
\text { Gln42 } \\
\text { Met82 } \\
\text { Tyr83 } \\
\text { Glu329 } \\
\text { Lys353 } \\
\text { Gly354 }\end{array}$ & $\begin{array}{l}\text { Ala475 } \\
\text { Asn487, } \\
\text { Phe456, Glu484, Tyr489, GIn493 } \\
\text { Leu455, Tyr453 } \\
\text { Gln493 } \\
\text { Tyr505 } \\
\text { Tyr449 } \\
\text { Thr500 } \\
\text { Gln498 } \\
\text { Phe486 } \\
\text { Gly496, Asn487, Tyr489 } \\
\text { Arg439 } \\
\text { Tyr505, Gly502 } \\
\text { Tyr505 }\end{array}$ \\
\hline 2 & rfhSP-D & S protein (open) & -20.63 & $\begin{array}{l}\text { Gln219 } \\
\text { His220 } \\
\text { Ala223 } \\
\text { Ser226 } \\
\text { Lys229 } \\
\text { Ser239 } \\
\text { Gly241 } \\
\text { Glu242 } \\
\text { Gln263 } \\
\text { Thr308 }\end{array}$ & $\begin{array}{l}\text { Gln493 } \\
\text { Tyr449 } \\
\text { Gln493, Phe490 } \\
\text { Ser494 } \\
\text { Tyr449 } \\
\text { Asn450 } \\
\text { Asn448, GIn498 } \\
\text { GIn498 } \\
\text { Arg346 } \\
\text { Arg466 }\end{array}$ \\
\hline 3 & ACE-2 & rfhSP-D & -24.30 & $\begin{array}{l}\text { Ser19 } \\
\text { Asp30 } \\
\text { Lys31 } \\
\text { His34 } \\
\text { Glu35 } \\
\text { Glu75 } \\
\text { Gly319 } \\
\text { Pro321 } \\
\text { Gln552 }\end{array}$ & $\begin{array}{l}\text { Ser328 } \\
\text { Thr255 } \\
\text { Thr305 } \\
\text { Gln258 } \\
\text { Pro307, Gly309 } \\
\text { Lys299 } \\
\text { Ala275 } \\
\text { Ala275 } \\
\text { Ala274, Tyr314 }\end{array}$ \\
\hline
\end{tabular}

Definition of abbreviations: ACE-2 = angiotensin-converting enzyme 2; rfhSP-D = recombinant fragment of human SP-D; S protein = Spike protein.

*The ACE2 residues in bold typeface interact with both S protein and rfhSP-D (docked structure).

tThe $\mathrm{S}$ protein residues in bold are predicted to be part of the common binding site for ACE-2 and rfhSP-D.

¥The structural coordinates of Phe486 are missing in the open conformation S protein (Protein Data Bank identification 6VYB).

\section{Results}

\section{rfhSP-D Interacts with the S Protein of SARS-CoV-2 and Human ACE-2 In Silico}

$S$ protein is known to interact via the RBM (aa 455-508) in the RBD (aa 319-527) with virus-binding hotspot residues comprising Lys31, Glu35, and Lys353 of dimeric hACE-2 $(15,16)$. The structure of hACE2 receptor, co-crystallized with S protein of SARS-CoV2 , is available in RCSB (pdb identification 6VW1). The receptor (ACE-2) and ligand (S protein) were separated and docked to validate the docking protocol. The redocked complex of ACE-2 and S protein had root mean square deviation of $7.9 \AA$. The close agreement between the docked and crystal structures validated the docking protocol used in this study.
In the case of docked solutions for $S$ protein and rfhSP-D (Figure E1 in the data supplement), the third-ranked docked pose with a binding energy of $-20.63 \mathrm{kcal} / \mathrm{mol}$ exhibited $\mathrm{rfhSP}-\mathrm{D}$ interactions with RBM residues Tyr449, Gln493, and Gln498, implying that rfhSP-D could bind to $S$ protein in a manner that can inhibit ACE-2-S protein interaction (Table 2 and Figure 1). To ascertain this hypothesis, the complex of $S$ protein with rfhSP-D was docked to ACE-2. S protein and rfhSP-D bound to ACE-2 via common interacting residues.

The top-ranked docked structure of ACE-2 and rfhSP-D had a binding energy of $-24.30 \mathrm{kcal} / \mathrm{mol}$. In this pose, rfhSP-D interacted with the virus-binding hotspot residues Ser19, Lys31, His34, and Glu35 of ACE-2, implying that rfhSP-D could bind to
ACE-2 in a manner that can inhibit ACE2-S protein interaction (Table 2 and Figure 1). To corroborate this postulate, the complex of ACE-2 with rfhSP-D was docked to $S$ protein. The top-ranked pose of ACE-2-rfhSP-D complex docked with open $S$ protein had a binding energy of -33.01 $\mathrm{kcal} / \mathrm{mol}$ and several common interactions between rfhSP-D and ACE-2 with S protein (Figure E1). The docking experiments led us to infer that rfhSP-D could bind to both ACE-2 as well as S protein and prevent ACE-2-S protein interaction.

\section{Single-Residue In Silico Mutants of rfhSP-D Displayed Lower Binding Affinities to S Protein and ACE-2} In silico mutational analysis was performed to evaluate the functional importance of residues of rfhSP-D that were identified to be 
A

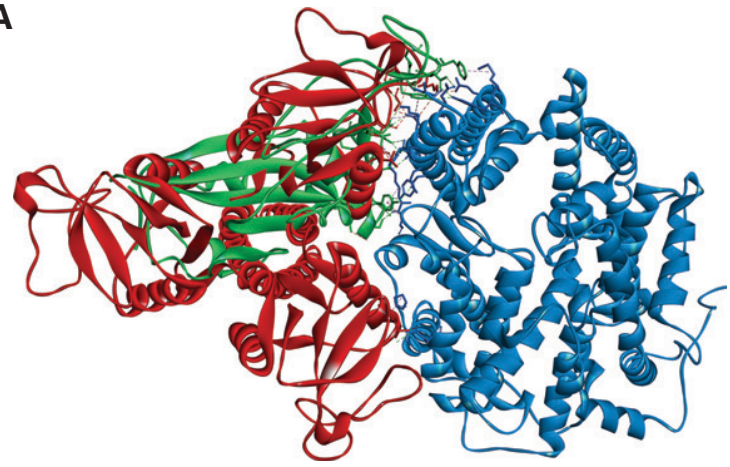

B

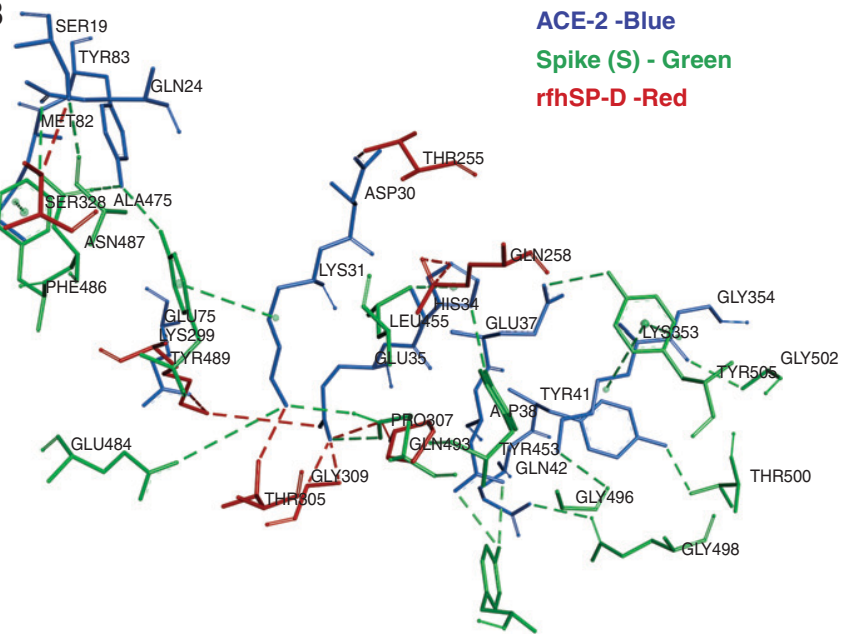

C
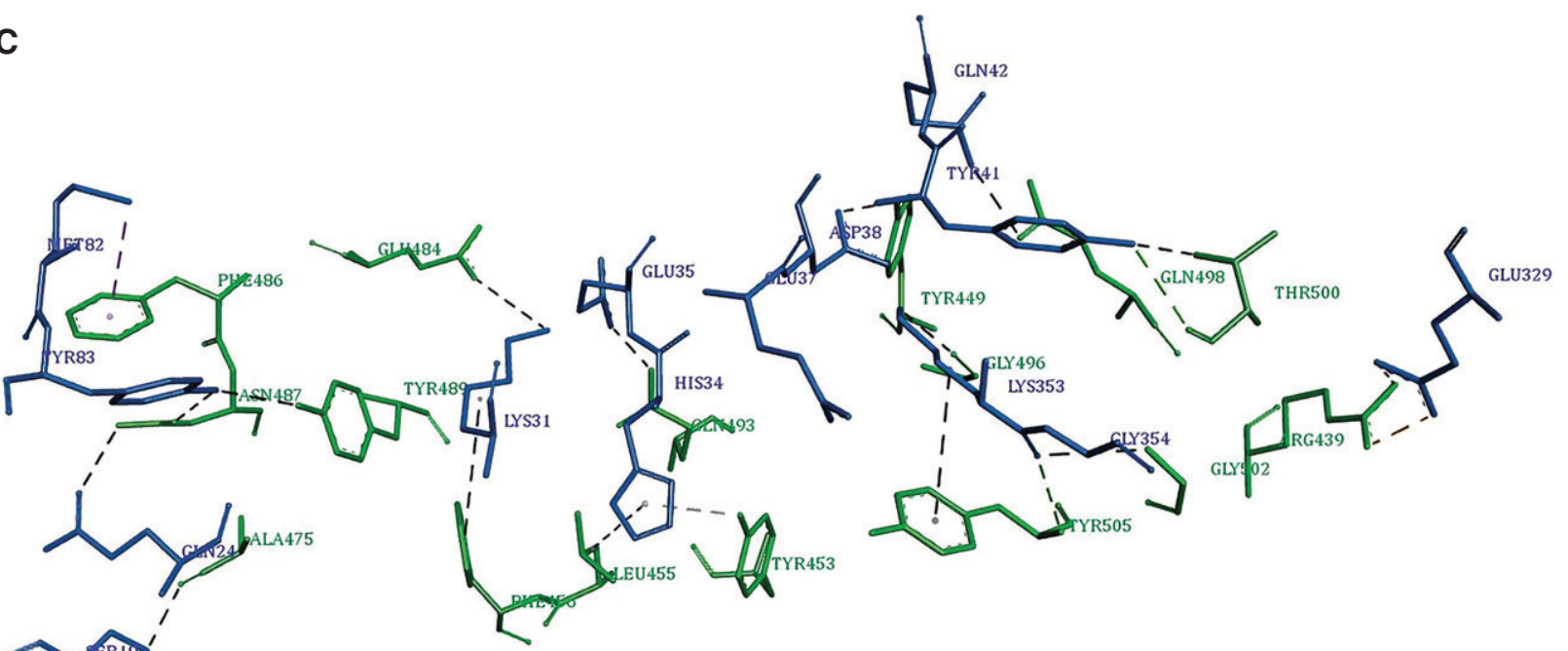

E
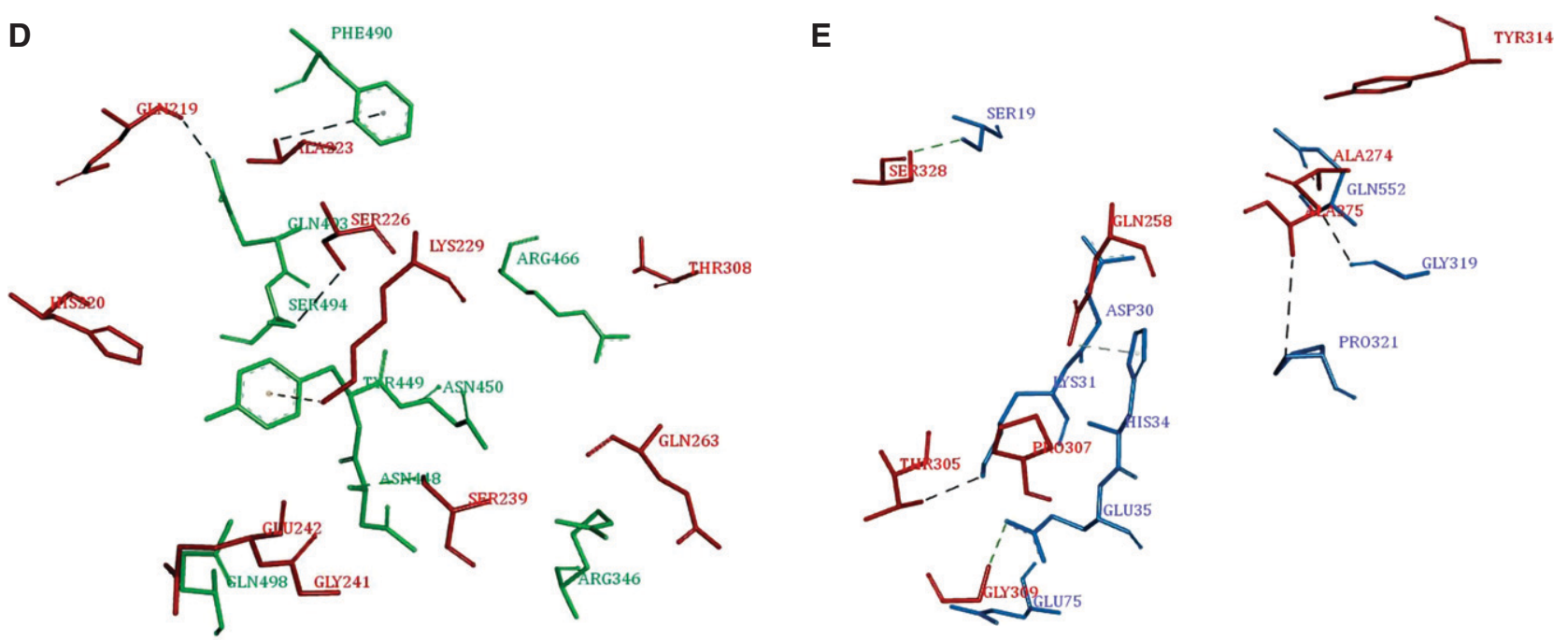

Figure 1. Tripartite interaction between spike protein (S protein) (green), recombinant fragment of human SP-D (surfactant protein D) (rfhSP-D) (red), and ACE-2 (angiotensin-converting enzyme 2) (blue) ( $A$ and B). ACE-2 residues Ser19, Lys31, Glu35 and His34 interact with both $S$ protein and rfhSP-D. The interactions between $S$ protein and ACE-2 are deduced from the crystal structure (Protein Data Bank identification 6VW1), and those between rfhSP-D and ACE-2 protein are based on docked complexes. $B$ is a zoomed view of $A$. Individual intermolecular interactions between $(C)$ S protein (green) and ACE-2 (blue), (D) S protein (green) and rfhSP-D (red), and ( $E$ ) rfhSP-D (red) and ACE-2 (blue). The S protein residues Tyr449, Gln493, and Gln498 participate in intermolecular interactions with both ACE-2 and rfhSP-D. 
involved in the intermolecular interactions with ACE-2 and S protein through docking studies (Table 2 and Figure 1). The rfhSP-D residues Ser328, Gly309, Pro307, Thr305, and Gln 258 of the ACE-2-rfhSP-D complex and Lys229, Glu242, Gly241, His220, and Gln219 of S protein-rfhSP-D complex were individually substituted to standard amino acids. All the mutants demonstrated lower binding affinities, except for substitutions with similar physicochemical properties (Figure 2), corroborating the importance of the mutated residues in intermolecular interactions.

\section{rfhSP-D Binds to the Immobilized S Protein of SARS-CoV-2 as Well as hACE-2}

The possible binding between rfhSP-D and $\mathrm{S}$ protein hinted by the docking analysis was confirmed in vitro via an indirect ELISA. rfhSP-D was found to bind the immobilized $S$ protein in a dose-dependent manner (Figure 3A). However, a significant difference in the absorbance was observed based on the specificity of the primary antibody used. $S$ protein-rfhSP-D binding that was probed with the polyclonal antibody against SP-D reported a significantly higher absorbance when compared with the wells that were probed with a monoclonal antibody directed against the CRD of SP-D. This difference suggests the involvement of CRD of rfhSP-D with the $S$ protein, and, therefore, the CRD was not available for interaction with the monoclonal antibody. $\mathrm{S}$ protein was also found to bind to the FL SP-D. The treatment of rfhSP-D with $10 \mathrm{mM}$ EDTA did not significantly alter the binding of rfhSP-D and FL SP-D to the $S$ protein (Figure $3 \mathrm{~B}$ ). Hence, rfhSP-D and FL SP-D bind to the $S$ protein in a dose-dependent, but calcium-independent, manner. A similar parallel experiment revealed that rfhSP-D bound ACE-2 in a dose-dependent manner (Figure 3C).

\section{rfhSP-D Inhibits the Interaction of S Protein and Its RBD with Biotinylated hACE-2 in a Calcium- Independent Manner}

Because rfhSP-D was found to bind to the $S$ protein as well as ACE-2, and both rfhSP-D and ACE- 2 were predicted to share the same binding site on the S protein, rfhSPD-mediated inhibition of the interaction between the RBD of the $S$ protein of SARSCoV-2 and ACE-2 was assessed using a colorimetric ELISA.

The wells were coated with either the $S$ protein or its RBD domain that was preincubated with rfhSP-D followed by addition of biotinylated hACE- 2 . The functionality and the range of the assay were initially assessed by verifying whether the assay could detect the binding of hACE- 2 at concentrations of $0.12 \mu \mathrm{g} / \mathrm{ml}$ and $0.06 \mu \mathrm{g} /$ $\mathrm{ml}$. The binding occurred in a dose-

dependent manner, confirming that the assay could detect binding between $S$ protein or its RBD domain with hACE- 2 at a

concentration as low as $60 \mathrm{ng} / \mathrm{ml}$ (Figure E2). A decrease in binding between the $S$ protein and hACE- 2 was observed as the

concentration of rfhSP-D increased (Figures 4 and 5). Approximately 50\% decrease in S protein-hACE-2 binding was observed as rfhSP-D concentration increased fivefold (Figure 4A). A similar result was observed between the binding of the RBD of $S$ protein and hACE-2. An eightfold increase in the concentration of rfhSP-D was found to
A

\begin{tabular}{|c|c|c|c|c|c|c|c|c|c|c|}
\hline AA Residues & GLN & 258 & THF & 305 & & 307 & & 309 & SEI & 3328 \\
\hline ALA & $\sqrt{b}$ & -0.596 & $\sqrt{b}$ & -1.054 & $\sqrt{3}$ & -1.071 & $\sqrt{b}$ & -0.646 & $\sqrt{b}$ & -0.075 \\
\hline ARG & $\sqrt{b}$ & -0.062 & 令 & 0.324 & $\Delta$ & -1.086 & $\sqrt{3}$ & -0.644 & 办 & 0.303 \\
\hline ASN & $\sqrt{b}$ & -0.234 & $\sqrt{4}$ & -0.964 & $\sqrt{b}$ & -0.393 & $\sqrt{b}$ & -0.43 & 办 & 0.06 \\
\hline ASP & 潒 & 0.386 & $\sqrt{b}$ & -1.058 & $\sqrt{4}$ & -0.521 & $\sqrt{b}$ & -0.239 & 象 & 0.241 \\
\hline CYS & $\sqrt{b}$ & -0.643 & $\sqrt{5}$ & -1.024 & $\sqrt{3}$ & -1.061 & $\sqrt{b}$ & -0.753 & $\sqrt{ }$ & -0.201 \\
\hline GLN & & 0 & & 0.764 & 㐿 & -0.547 & $\sqrt{3}$ & -0.37 & $\sqrt{4}$ & -0.005 \\
\hline GLU & 1) & 0.849 & $\sqrt{b}$ & -0.642 & $\sqrt{b}$ & -0.28 & $\sqrt{b}$ & -0.115 & 武 & 0.228 \\
\hline GLY & $\sqrt{b}$ & -0.492 & & -1.085 & $\sqrt{b}$ & -1.031 & & & $\sqrt{b}$ & -0.127 \\
\hline HIS & $\sqrt{b}$ & -0.07 & $\sqrt{b}$ & -0.103 & $\sqrt{3}$ & -1.356 & $\sqrt{b}$ & -0.196 & 価 & 0.203 \\
\hline ILE & $\sqrt{b}$ & -0.233 & $\sqrt{b}$ & -0.675 & $\sqrt{b}$ & -0.291 & $\sqrt{b}$ & -0.873 & $\sqrt{b}$ & -0.125 \\
\hline LEU & $\sqrt{b}$ & -0.4 & $\sqrt{4}$ & -0.614 & $\sqrt{3}$ & -0.491 & $\sqrt{b}$ & -0.886 & $\sqrt{3}$ & -0.073 \\
\hline LYS & 急 & 0.325 & $\sqrt{3}$ & -0.537 & $\sqrt{3}$ & -0.628 & $\sqrt{3}$ & -0.519 & 価 & 0.169 \\
\hline MET & $\sqrt{b}$ & -0.338 & $\sqrt{b}$ & -0.586 & $\sqrt{b}$ & -1.103 & $\sqrt{b}$ & -0.81 & $\sqrt{b}$ & -0.015 \\
\hline PHE & $\sqrt{3}$ & -0.441 & $\sqrt{4}$ & -0.147 & $\sqrt{3}$ & -0.453 & $\sqrt{b}$ & -0.505 & $\sqrt{b}$ & -0.113 \\
\hline PRO & $\sqrt{b}$ & -0.785 & & -1.041 & & & $\sqrt{3}$ & -0.931 & $\sqrt{4}$ & -0.159 \\
\hline SER & $\sqrt{ }$ & -0.301 & $\sqrt{3}$ & -0.94 & $\sqrt{b}$ & -0.174 & $\sqrt{3}$ & -0.494 & & U \\
\hline THR & $\sqrt{b}$ & -0.452 & & 0 & $\sqrt{3}$ & -0.234 & $\sqrt{b}$ & -0.66 & 像 & 0.078 \\
\hline TRP & $\sqrt{b}$ & -0.305 & $\sqrt{b}$ & -0.289 & $\sqrt{4}$ & -0.376 & $\sqrt{b}$ & -0.388 & 象 & 0.218 \\
\hline TYR & $b$ & -0.317 & a & 0.516 & 4 & -0.779 & $\sqrt{3}$ & -0.426 & 斥 & 0.029 \\
\hline AL & $\sqrt{h}$ & -0.322 & $\sqrt{2}$ & -0.151 & & 0.828 & $\sqrt{b}$ & -0.602 & $\sqrt{b}$ & -0.125 \\
\hline
\end{tabular}

B

\begin{tabular}{|c|c|c|c|c|c|c|c|c|}
\hline AA Residues & ${ }^{s}$ GLI & 1219 & 220 & & 229 & GLY & 241 & GLU242 \\
\hline ALA & $\sqrt{5}$ & $-0.584 \sqrt{b}$ & -0.207 & $\sqrt{b}$ & -1.602 & $\sqrt{b}$ & -0.628 & $\sqrt{y} \quad-0.467$ \\
\hline ARG & 闪 & 0.274 & 0.408 & $\sqrt{b}$ & -0.625 & $\sqrt{b}$ & -0.888 & -0.506 \\
\hline ASN & $\sqrt{b}$ & $-0.504 \sqrt{b}$ & -0.348 & $\sqrt{4}$ & -1.396 & $\sqrt{b}$ & -0.672 & -0.512 \\
\hline ASP & $\sqrt{b}$ & $-0.242 \sqrt{b}$ & -0.666 & $\sqrt{4}$ & -1.646 & $\sqrt{b}$ & -0.788 & -0.12 \\
\hline CYS & $\sqrt{b}$ & $-0.862 \sqrt{b}$ & -0.435 & $\sqrt{b}$ & -1.423 & $\sqrt{b}$ & -0.879 & -0.49 \\
\hline GLN & & $0 \sqrt{4}$ & -0.322 & $\sqrt{b}$ & -0.681 & $\sqrt{b}$ & -0.814 & -0.488 \\
\hline GLU & $\sqrt{b}$ & $-0.043 \sqrt{b}$ & -0.572 & $\sqrt{b}$ & -1.235 & $\sqrt{4}$ & -0.669 & 0 \\
\hline GLY & $\sqrt{5}$ & $-1.008 \sqrt{b}$ & -0.652 & $\sqrt{4}$ & -1.638 & & & -0.418 \\
\hline HIS & $\sqrt{b}$ & -0.32 & & 迎 & 0.556 & $\sqrt{b}$ & -0.596 & 144 \\
\hline ILE & $\sqrt{b}$ & -0.539 答 & 0.416 & $\sqrt{b}$ & -0.786 & $\sqrt{b}$ & -0.878 & $\sqrt{y} \quad-0.231$ \\
\hline LEU & $\sqrt{b}$ & $-0.098 \sqrt{b}$ & -0.361 & $\sqrt{b}$ & -0.74 & $\sqrt{b}$ & -0.917 & -0.246 \\
\hline LYS & $\sqrt{b}$ & $-0.919 \widehat{A}$ & 0.447 & & & $\sqrt{b}$ & -0.832 亿 & 0.195 \\
\hline MET & $\sqrt{b}$ & $-0.168 \sqrt{b}$ & -0.249 & $\sqrt{b}$ & -1.387 & $\sqrt{b}$ & -0.797 & $\begin{array}{ll}y & -0.237\end{array}$ \\
\hline PHE & $\sqrt{b}$ & $-0.489 \sqrt{b}$ & -0.04 & $\sqrt{2}$ & -0.306 & $\sqrt{b}$ & -0.404 亿 & 0.03 \\
\hline PRO & $\sqrt{b}$ & $-0.575 \sqrt{b}$ & -0.768 & $\sqrt{3}$ & -1.375 & $\sqrt{b}$ & -0.911 & 1.008 \\
\hline SER & $\sqrt{b}$ & $-0.651 \sqrt{b}$ & -0.23 & $\sqrt{b}$ & -1.701 & $\sqrt{4}$ & -0.124 & -0.4 \\
\hline THR & $\sqrt{ }$ & $-0.544 \sqrt{b}$ & -0.302 & $\sqrt{4}$ & -1.426 & $\sqrt{ }$ & -0.481 & -0.521 \\
\hline TRP & $\sqrt{b}$ & -0.439 命 & 0.005 & $\sqrt{b}$ & -0.029 & $\sqrt{ }$ & $-0.405 \widehat{1}$ & 0.199 \\
\hline TYR & $\sqrt{b}$ & $-0.407 \sqrt{b}$ & -0.059 & $\sqrt{b}$ & -0.083 & $\sqrt{b}$ & $-0.513 \widehat{4}$ & 0.104 \\
\hline VAL & $\sqrt{b}$ & $-0.496 \sqrt{b}$ & -0.02 & $\sqrt{b}$ & -1.178 & 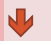 & -0.773 & -0.342 \\
\hline
\end{tabular}

Figure 2. Heatmap representation of effect of single-residue mutations of rfhSP-D ( $y$ axis) on binding energy of docked rfhSP-D complexed with $(A)$ ACE-2 and $(B)$ S protein using mCSM-PPI2 web server. Each of the rfhSP-D residues involved in interaction with virus-binding hotspot of ACE-2 and the receptor-binding motif of the S protein were mutated to the standard amino acids, and its effect on binding energy of the complex was assessed. Most of the mutations led to decrease in the binding affinity (red cells). Few of the conserved substitutions led to increase in the binding affinity (blue cells), highlighting the likely functional importance of the mutated residues. 

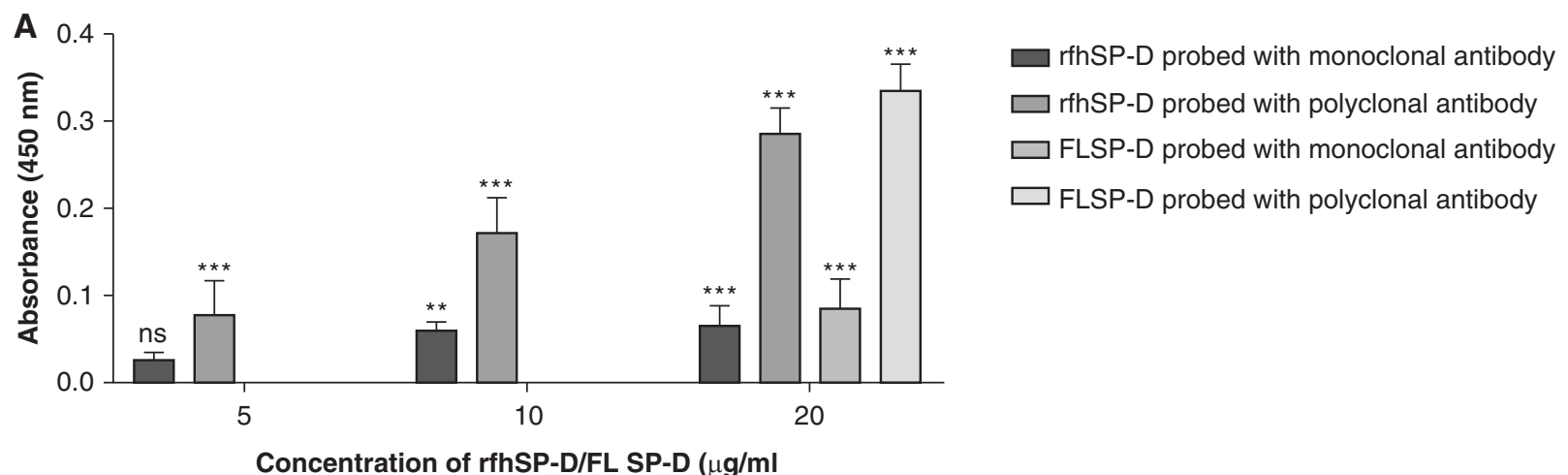

Concentration of rfhSP-D/FL SP-D $(\mu \mathrm{g} / \mathrm{ml}$

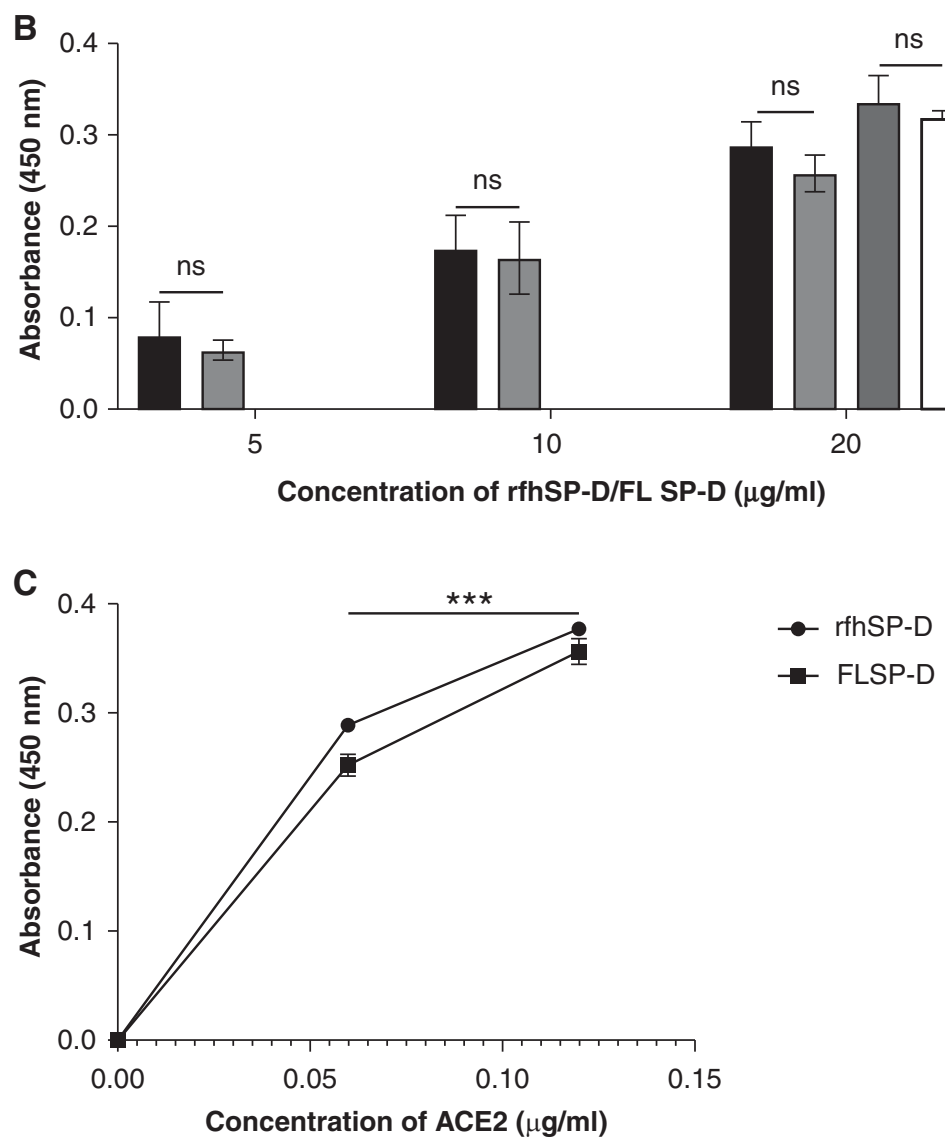

Figure 3. rfhSP-D binds to the immobilized S protein of severe acute respiratory syndrome coronavirus-2 (SARS-CoV-2); immobilized rfhSP-D binds to human ACE-2 (hACE-2) in a dose-dependent, but calcium-independent, manner. ELISA showing binding of rfhSP-D to the immobilized S protein. Microtiter wells were coated with $0.3 \mu \mathrm{g} / \mathrm{ml}(0.54 \mathrm{nM})$ of S protein. rfhSP-D (20, 10, and $5 \mu \mathrm{g} / \mathrm{ml}$ or $0.334,0.167$, and $0.083 \mu \mathrm{M}$ in PBS with $5 \mathrm{mM} \mathrm{CaCl}_{2}$ ) were added to the wells. Full-length (FL) SP-D $(20 \mu \mathrm{g} / \mathrm{ml}$ or $0.038 \mu \mathrm{M})$ was also used in a similar manner. BSA (20 $\left.\mu \mathrm{g} / \mathrm{ml}\right)$ was used as a non-specific protein control (mean of the normalized triplicates \pm SEM $=0.07 \pm 0.006$ with polyclonal antibody and $0.025 \pm$ 0.006 with monoclonal antibody). (A) S protein-SP-D binding was detected with either polyclonal or monoclonal antibodies against SP-D. (B) To assess the effect of calcium in the SP-D-S protein interaction, rfhSP-D (20, 10, and $5 \mu \mathrm{g} / \mathrm{ml}$ or 0.334, 0.167, and 0.083 $\mu \mathrm{M})$ and FL SP-D (20 $\mu \mathrm{g} / \mathrm{ml}$ or $0.038 \mu \mathrm{M})$, either with or without $10 \mathrm{mM}$ EDTA, was used in a similar manner and probed with polyclonal antibodies against SP-D. (C) The binding of immobilized rfhSP-D to hACE-2 was assessed by coating microtiter wells with $0.1 \mu \mathrm{g} / \mathrm{ml}$ of FL SP-D (1.9 nM) or rfhSP-D (16.7 $\mathrm{nM})$. BSA $(0.1 \mu \mathrm{g} / \mathrm{ml})$ was used as a non-specific protein control (mean of the normalized triplicates $\pm \mathrm{SEM}=0.006 \pm 0.005)$. Decreasing concentration of hACE-2 $(0.12,0.06$, and $0.00 \mu \mathrm{g} / \mathrm{ml}$ or $0.52,0.26$, and $0.0 \mathrm{nM})$ was added to the wells. The SP-D-hACE-2 binding was detected using streptavidin-HRP. The background was subtracted from all data points. The data were expressed as the mean of triplicates \pm SD. Significance was determined using the two-way ANOVA $(n=3)$ test. ${ }^{\star \star} P<0.05$ and ${ }^{\star \star \star} P<0.0001$. ns $=$ no significance. 
A
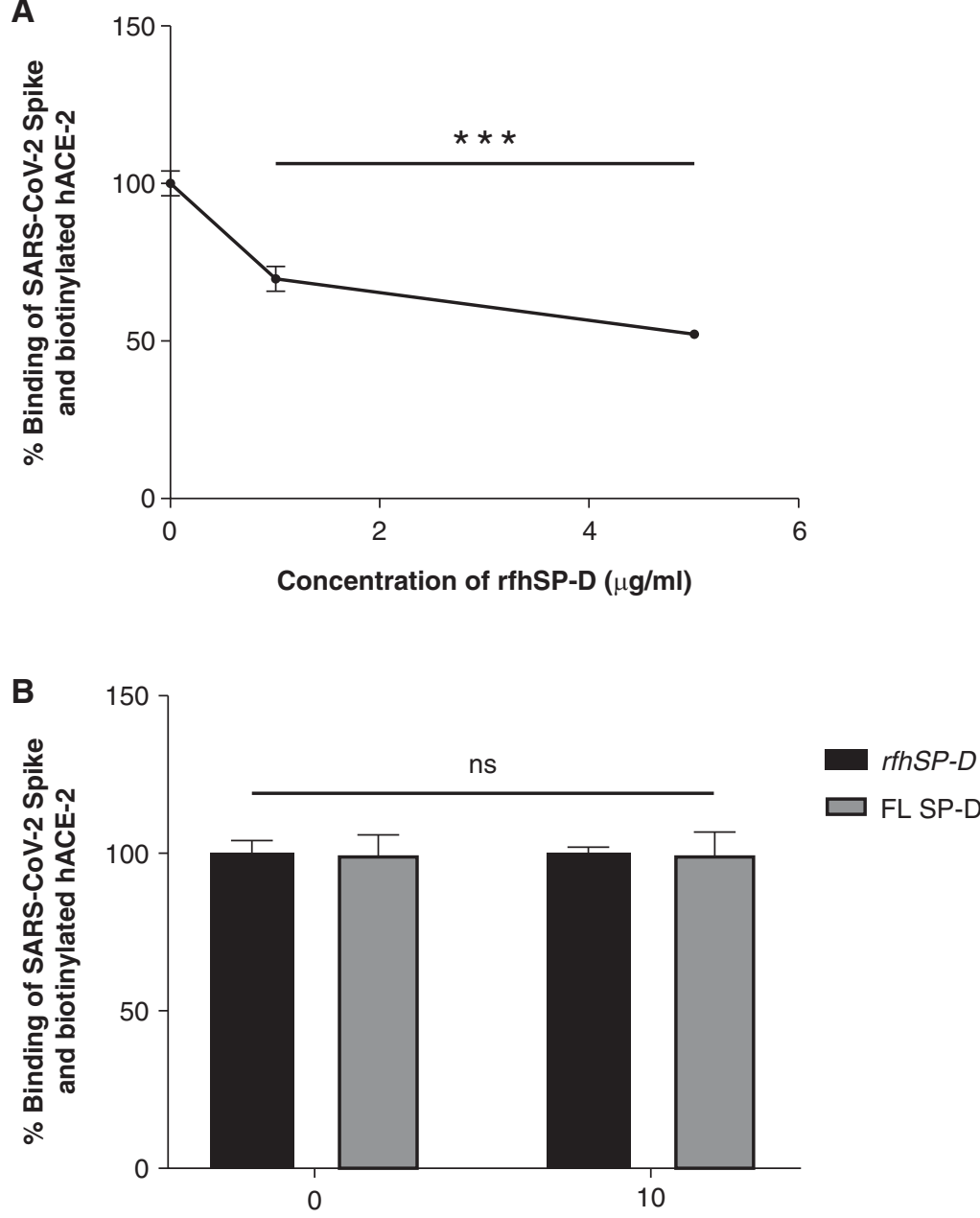

Concentration of EDTA ( $\mathrm{mM})$

Figure 4. rfhSP-D inhibits the interaction between S protein of SARS-CoV-2 and biotinylated hACE-2 in a calcium-independent manner $(A$ and $B)$. Microtiter wells were coated with 0.3 $\mu \mathrm{g} / \mathrm{ml}(0.54 \mathrm{nM})$ of S protein. (A) After blocking, rfhSP-D (5, 1, and $0 \mu \mathrm{g} / \mathrm{ml}$ or 83.5, 16.7, and $0.0 \mathrm{nM}$ ) was added and incubated for 1 hour, followed by probing with biotinylated hACE-2. $(B)$ To assess the effect of calcium in the SP-D-mediated inhibition of S protein-hACE-2 interaction, $5 \mu \mathrm{g} / \mathrm{ml}$ or $83.5 \mathrm{nM}$ of rfhSP-D and FL SP-D $(5 \mu \mathrm{g} / \mathrm{ml}$ or $9.5 \mathrm{nM})$ with or without 10 $\mathrm{mM}$ EDTA was added. BSA $(5 \mu \mathrm{g} / \mathrm{ml}$ ) was used as nonspecific protein control (mean of the normalized triplicates \pm SEM $=0.297 \pm 0.005$ ). S protein-hACE-2 binding was detected with streptavidin-HRP. Background was subtracted from all data points. The data were normalized with $100 \% \mathrm{~S}$ protein-hACE-2 binding being defined as the mean of the absorbance recorded from the control sample $(0 \mu \mathrm{g} / \mathrm{ml}$ rfhSP-D). The data were presented as the mean of the normalized triplicates \pm SEM. Significance was determined using the one-way ANOVA $(n=3)$. ${ }^{\star * \star} P<0.0001$

decrease RBD-hACE-2 interaction by 25\% (Figure 5A). No significant difference was observed when binding experiments were carried out with and without $10 \mathrm{mM}$ EDTA in terms of rfhSP-D-mediated S protein/ RBD-hACE-2 interaction (Figures $4 \mathrm{~B}$ and 5B). Hence, rfhSP-D-mediated inhibition of the interaction between the RBD of the $S$ protein or the $\mathrm{S}$ protein itself with biotinylated hACE2 occurred in a calcium-independent manner.

\section{rfhSP-D Treatment Inhibits SARS- CoV-2 Infection and Replication}

As rfhSP-D is known to induce apoptosis in cancer and immortalized cells (22, 24-26), the effect of rfhSP-D on Vero cells was assessed using an MTT assay. rfhSP-D treatment had no significant effect on the viability of Vero cells (Figure E3). At the outset, the $\mathrm{TCID}_{50}$ values of the clinical samples were obtained by evaluating the cytopathic effects using an MTT assay. As expected, when Vero cells were challenged with $100 \mathrm{TCID}_{50}$ or $50 \mathrm{TCID}_{50}$ of viral samples from SARS-CoV-2 clinical samples, a $50 \%$ or $25 \%$ reduction in cell viability was observed, respectively, compared with the viability of uninfected Vero cells, confirming the assayed $\mathrm{TCID}_{50}$ values (Figure 6). The control samples showed no significant difference in the cell viability from the uninfected Vero cells when the control sample volumes equivalent to $100 \mathrm{TCID}_{50}$ and $50 \mathrm{TCID}_{50}$ of the matched clinical cases were used.

The effect of rfhSP-D on the replication of SARS-CoV-2 (100 TCID T0 $_{\text {/well; }}$ MOI, 0.01 ) in Vero cells was evaluated by measuring the concentrations of the $R d R p$ gene of SARS-CoV-2 by real-time qPCR 24 hours after infection. Pretreatment of the positive samples $(n=15)$, comprising SARSCoV-2 with rfhSP-D, led to a reduction in $\mathrm{RdRp}$ expression in a dose-dependent manner (Figure 7 and Table E1). The pretreatment of samples from all categories of SARS-CoV-2-positive cases (as representatives, the Figure 7 shows the data for $1 \mathrm{~S}$ [category 2] and 3S [category 6]) with $0.835 \mu \mathrm{M}$ rfhSP-D led to $\sim 4.5$-fold reduction $(-4.5 \log 2)$ of $R d R p$ transcript compared with the untreated positive sample-challenged Vero cells. There was no significant difference in the Ct values of the $R d R p$ gene from the untreated and control sample-treated Vero cells. Similarly, pretreatment with $1.67 \mu \mathrm{M}$ rfhSP-D resulted in $\sim 5.5$-fold reduction $(-5.5 \log 2)$ of $\mathrm{RdRp}$ mRNA expression. Remdesivir, one of the antiviral drugs proposed for COVID-19, which functions by inhibiting viral RNA synthesis, was found to inhibit SARS-CoV-2 replication by $\sim 4$-fold $(-4 \log 2)$. Hence, rfhSP-D blocked SARS-CoV-2 infection in addition to inhibiting the replication of SARS-CoV-2 significantly better than remdesivir at both tested concentrations (0.835 $\mu \mathrm{M}$ and $1.67 \mu \mathrm{M}$ rfhSP-D).

As rfhSP-D was found to interact with $S$ protein and ACE-2, proteins that play an integral role in viral host cell recognition and entry, the role of rfhSP-D in viral infectivity was assessed in a similar manner to replication. Vero cells infected with SARSCoV-2-positive samples $\left(500\right.$ TCID $_{50} /$ well; MOI, 0.05) showed an rfhSP-D dosedependent decrease in the expression levels of the RdRp gene 2 hours after infection (Figure 8 and Table E2). Clinical samples from all the categories of patients with 

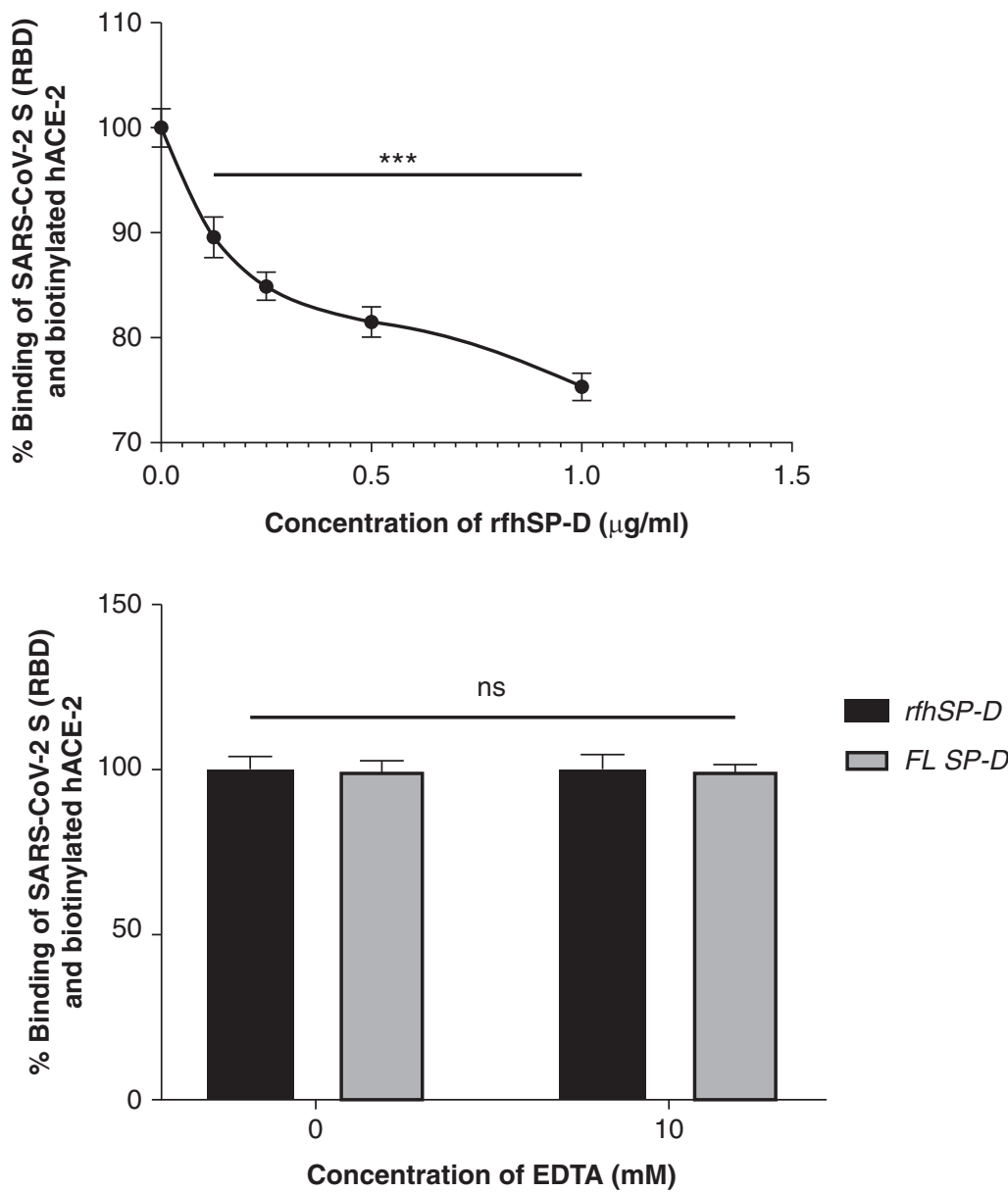

Figure 5. rfhSP-D inhibits the interaction between the receptor-binding domain (RBD) of the $S$ protein of SARS-CoV-2 and biotinylated hACE-2 in a calcium-independent manner. Microtiter wells were coated with $0.1 \mu \mathrm{g} / \mathrm{ml}(2.5 \mathrm{nM})$ of $S$ protein RBD. After blocking, decreasing concentrations of rfhSP-D $(1,0.5,0.25,0.125$, and $0 \mu \mathrm{g} / \mathrm{ml}$ or $16.7,8.35,4.18$, and $2.09 \mathrm{nM}$ in PBS with $\left.5 \mathrm{mM} \mathrm{CaCl}_{2}\right)(A)$ were incubated for 1 hour, followed by probing with biotinylated hACE-2. To assess the effect of calcium in the rfhSP-D-mediated inhibition of $S$ protein RBD-hACE-2 interaction $(B), 5 \mu \mathrm{g} / \mathrm{ml}(83.5 \mathrm{nM})$ of rfhSP-D or FL SP-D $(5 \mu \mathrm{g} / \mathrm{ml}$ or $9.5 \mathrm{nM})$ with or without $10 \mathrm{mM}$ EDTA was used. BSA $(5 \mu \mathrm{g} / \mathrm{ml})$ was used as a nonspecific protein control (mean of the normalized triplicates \pm SEM $=0.894 \pm 0.006$ [this data is not plotted in the figure]). S protein RBD-hACE-2 binding was detected using streptavidin-HRP. Background was subtracted from all data points. The data obtained were normalized with $100 \% \mathrm{~S}$ protein RBD-hACE-2 binding being defined as the mean of the absorbance recorded from the control sample $(0 \mu \mathrm{g} / \mathrm{ml} \mathrm{rfhSP}-\mathrm{D})$. The data were presented as the mean of the normalized triplicates \pm SEM. Significance was determined using the one-way ANOVA $(n=3)$. ${ }^{\star \star \star} P<0.0001$

SARS-CoV-2 (as representatives, Figure 8 shows the data from $2 S$ [category 6 ] and 9AS [catagory 5a]) showed $\sim 1.25$-fold reduction $(-1.25 \log 2)$ or $\sim 2$-fold reduction $(-2 \log 2)$ in $R d R p$ gene expression with the samples pretreated with either $0.835 \mu \mathrm{M}$ or $1.67 \mu \mathrm{M}$, respectively, of rfhSP-D.

Remdesivir was used as a control (remdesivir does not inhibit SARS-CoV-2 infection). Thus, pretreatment of SARS-CoV-2 in the clinical sample with rfhSP-D appears to make $S$ protein unavailable to interact with the ACE-2 receptor on the host cell, thus reducing the infectivity of the virus and subsequent viral replication in a dosedependent manner.

\section{Discussion}

The present study explored the likely protective effect of rfhSP-D against SARS$\mathrm{CoV}-2$. As predicted by the docking study, rfhSP-D interacted with the S protein of
SARS-CoV-2, its RBD, and ACE-2. Importantly, these interactions appear to have contributed to significant inhibition of infectivity and replication of SARS-CoV-2 present in the clinical samples derived from patients with asymptomatic, symptomatic, and severe COVID-19 (Figure 9).

One of the first steps of the SARS-CoV2 infection is the binding of the $S$ protein to the host cell via the ACE-2 receptor (27). S1 protein is known to interact with the ACE-2 receptor via the RBM (455-508) in the RBD (aa 319-527), with virus-binding hotspot residues comprising Lys31, Glu35, and Lys353 of dimeric ACE-2 (15, 28, 29). Previously, SP-D interaction with the S protein of SARS-CoV, which shares 74\% homology with the RBD of SARS-CoV-2 (12), has been reported. rfhSP-D is also known to bind to viral surface proteins such as hemagglutinin and neuraminidase of influenza A virus, gp120 of human immunodeficiency virus $1(7,30)$, and $S$ protein of SARS-CoV (12). Thus, the possibility of rfhSP-D binding to the $S$ protein of SARS-CoV-2 was examined.

In silico interaction of $\mathrm{rfhSP}-\mathrm{D}$ with RBD of the S protein of SARS-CoV-2 revealed that Tyr449, Gln493, and Gln498 of RBD overlapped with the residues that are essential for the binding of the $\mathrm{S}$ protein to the target protein ACE-2. The binding of the $S$ protein to rfhSP-D or FL SP-D was confirmed using an indirect ELISA. A comparatively lower absorbance with the monoclonal antibodies raised against the CRD region of human SP-D than the polyclonal antibodies could be attributed to the fact that the binding between rfhSP-D and the $S$ protein occurred through the CRD region of rfhSP-D and FL SP-D.

Furthermore, calcium independence suggested an involvement of protein-protein interaction. A significant inhibition of the $S$ protein-ACE-2 interaction in presence of rfhSP-D suggested that rfhSP-D could interfere with the binding of the SARS-CoV2 to the host cell, an essential step for infection to occur.

Clinical samples of SARS-CoV-2 were used to assess whether rfhSP-D modulated the infectivity and replication of the virus (isolation of the virus in the laboratory conditions may introduce alterations) in vitro. For assessing replication, qRT-PCR of the $R d R p$ gene, which is essential for the replication of viral RNA, was measured. Remdesivir was used as a positive control for replication inhibition. Remdesivir, an 


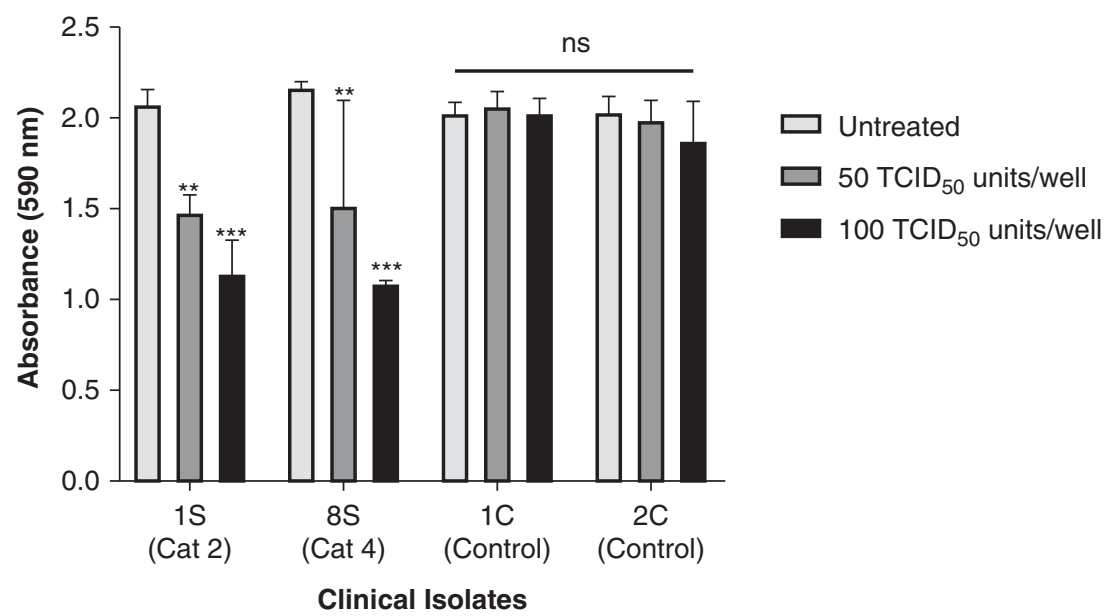

Figure 6. Determination of $50 \%$ tissue culture infective dose $\left(\mathrm{TCID}_{50}\right)$ value of the clinical samples in Vero cells using an MTT assay. Vero cells $\left(5 \times 10^{4} /\right.$ well) were seeded in complete MEM in 96-well culture plates and grown overnight at $37^{\circ} \mathrm{C}$ with $5 \% \mathrm{CO}_{2}$. Swab samples of 15 confirmed cases of coronavirus disease (COVID-19), including symptomatic contacts of laboratory-confirmed cases (category [Cat] 2) $(n=2)$, hospitalized patients with severe acute respiratory infections (Cat 4$)(n=3)$, asymptomatic direct and high-risk contacts of laboratory-confirmed cases (Cat 5a) $(n=7)$, and hospitalized patients with symptomatic influenza-like illness (Cat 6$)(n=3)$ who had tested positive by RT-PCR test for SARS-CoV-2, and 15 controls (at different dilutions/well) were added to the cells and incubated for 1 hour. The supernatants were removed, and the wells were washed twice with sterile PBS. Fresh complete MEM was added to the wells, and the cells were incubated for 96 hours. Viability of the cells was evaluated using an MTT assay. MTT $(0.5 \mathrm{mg} / \mathrm{ml})$-containing medium was added to the wells for 4 hours. The supernatants were removed, and cells were lysed using DMSO. Absorbance was measured at $590 \mathrm{~nm}$. The data obtained were normalized with $100 \%$ cell viability being defined as the mean of the absorbance recorded from the control sample $\left(0 \mathrm{TCID}_{50} / \mathrm{well}\right)$, and TCID $\mathrm{D}_{50}$ units were evaluated in each sample. The same assay was used to validate the cytopathic effects of $100 \mathrm{TCID}_{50}$ and 50 TCID 50 units of the samples. Data for cytopathic effects of $100 \mathrm{TCID}_{50}$ units for all 15 cases and 15 controls have been provided in the Table E1. The representative data for cases $(n=2)$ and controls $(n=2)$ are presented as the mean of the normalized triplicates \pm SEM. Significance was determined using the twoway ANOVA $(n=3)$ test. ${ }^{* *} P<0.01$ and ${ }^{* * *} P<0.0001$

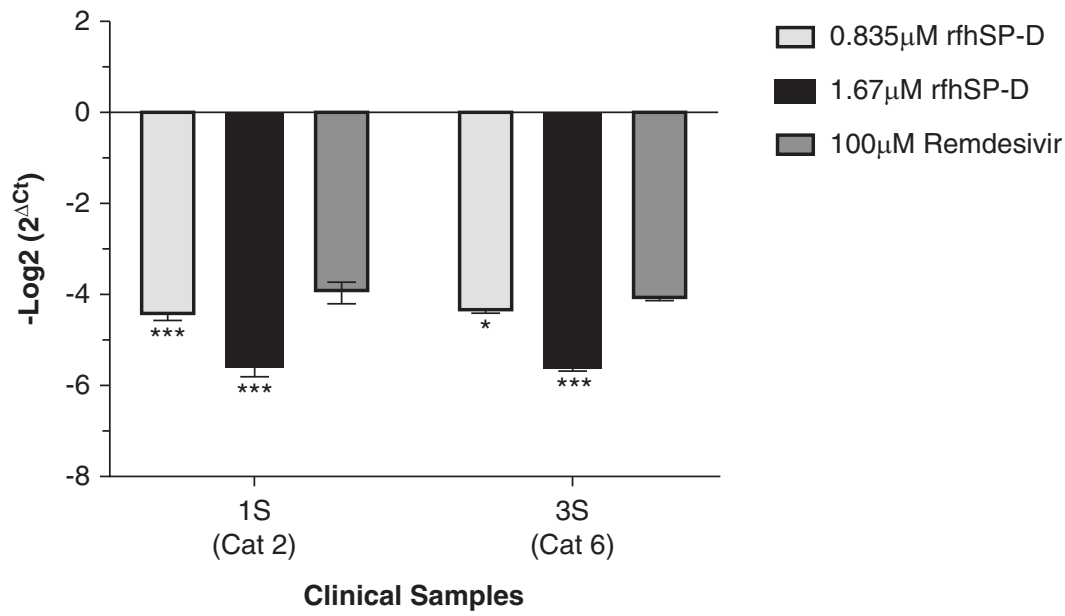

Figure 7. rfhSP-D pretreatment of SARS-CoV-2 significantly inhibited its replication. Vero cells $\left(5 \times 10^{4} /\right.$ well $)$ were seeded in complete MEM in 96-well culture plates and grown overnight at $37^{\circ} \mathrm{C}$ under $5 \% \mathrm{CO}_{2}$. Cells were washed with sterile PBS twice. SARS-CoV-2 clinical samples (100 TCID $50 /$ well; $\mathrm{MOI}$ 0.01), including symptomatic contacts of laboratory-confirmed cases (Cat 2) $(n=2)$, hospitalized patients with severe acute respiratory infections (Cat 4$)(n=3)$, asymptomatic direct and high-risk contacts of laboratory-confirmed cases (Cat $5 a)(n=7)$, and hospitalized patients with symptomatic influenza-like illness (Cat 6$)(n=3)$ who had tested positive by RT-PCR test for SARS-CoV-2, were preincubated with rfhSP-D $(0 \mu \mathrm{g} / \mathrm{ml}, 50 \mu \mathrm{g} / \mathrm{ml}$, and $100 \mu \mathrm{g} / \mathrm{ml}$ or $0,0.835$, and $1.67 \mu \mathrm{M})$ in MEM containing $5 \mathrm{mM} \mathrm{CaCl}_{2}$ for 1 hour at room temperature. The pretreated or untreated virus in the sample was added to the cells and incubated for 1 hour at $37^{\circ} \mathrm{C}$ under $5 \% \mathrm{CO}_{2}$. The wells were washed with PBS twice, and infection medium (MEM + 0.3\% BSA) was added to the cells and incubated for 24 hours at $37^{\circ} \mathrm{C}$. The supernatants were collected, and RNA was extracted by Perkin Elmer automated extractor and subjected to qRT-PCR for SARS-CoV-2. For control samples, the volume of the sample taken was equivalent to the volume of the case sample $\left(100 \mathrm{TCID}_{50}\right)$ in which no RdRp expression was detected. The relative expression of RdRp was calculated using rfhSP-D-untreated cells ( $0 \mu \mathrm{M}$ rfhSP-D), infected with respective samples as the calibrator. Data are provided in Table E1, and data of representative cases $(n=2)$ are presented as the mean of triplicates $(n=3)$. Error bars represent \pm SEM. Significance (compared with $100 \mu \mathrm{M}$ remdesivir) was determined using the two-way ANOVA test. ${ }^{*} P<0.05$ and ${ }^{\star \star \star} P<0.0001$. 


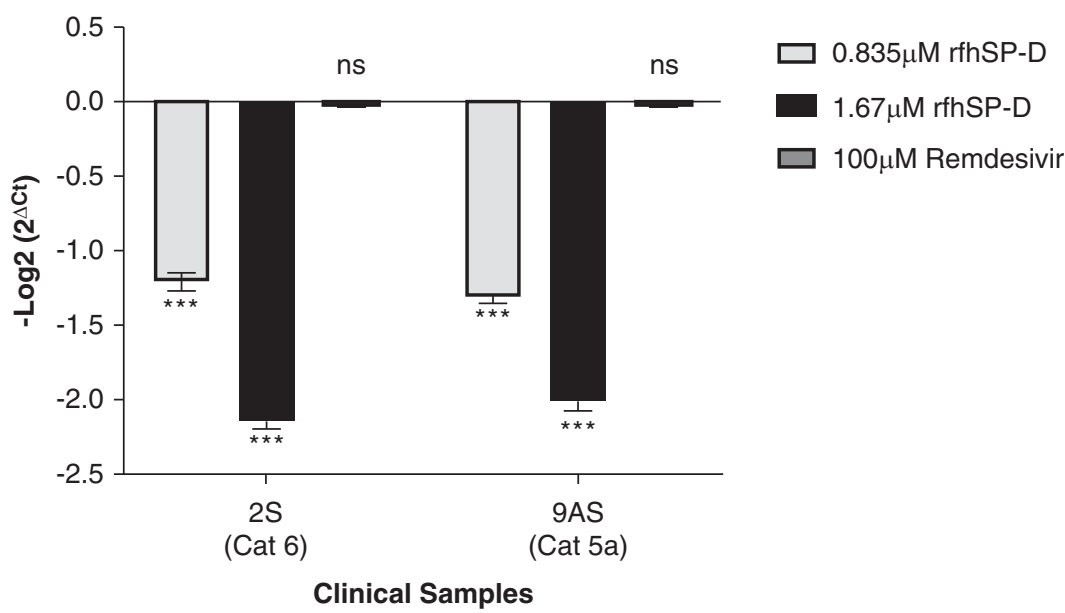

Figure 8. rfhSP-D pretreatment of SARS-CoV-2 significantly inhibited its infectivity. Vero cells $\left(5 \times 10^{5} /\right.$ well) were seeded in complete MEM in 12 -well culture plates and grown overnight at $37^{\circ} \mathrm{C}$ under $5 \% \mathrm{CO}_{2}$. Cells were washed with sterile PBS twice. SARS-CoV-2 clinical samples $(500$ $\mathrm{TCID}_{50} /$ well, multiplicity of infection, 0.05), including symptomatic contacts of laboratory-confirmed cases $($ Cat 2) $(n=2)$, hospitalized patients with severe acute respiratory infections (Cat 4$)(n=3)$, asymptomatic direct and high-risk contacts of laboratory-confirmed cases (Cat $5 a)(n=$ 7 ), and hospitalized patients with symptomatic influenza-like illness (Cat 6) $(n=3)$ who had tested positive by RT-PCR test for SARS-CoV-2, were preincubated with rfhSP-D $(0 \mu \mathrm{g} / \mathrm{ml}, 50 \mu \mathrm{g} / \mathrm{ml}$, and $100 \mu \mathrm{g} / \mathrm{ml}$ or $0,0.835$, and $1.67 \mu \mathrm{M})$ in MEM containing $5 \mathrm{mM} \mathrm{CaCl} \mathrm{f}_{2}$ for 1 hour at room temperature and 1 hour at $4^{\circ} \mathrm{C}$. This pretreated or untreated virus-containing sample was added to the cells and incubated for 1 hour at $37^{\circ} \mathrm{C}$ under $5 \% \mathrm{CO}_{2}$. The wells were washed with PBS twice, infection medium (MEM $+0.3 \% \mathrm{BSA}$ ) was added to the cells and incubated for 2 hours at $37^{\circ} \mathrm{C}$ under $5 \% \mathrm{CO}_{2}$. The cells were scraped, and the media containing scraped cells were collected. RNA was extracted and subjected to RT-PCR for SARS-CoV-2. For control samples, the volume of the sample taken was equivalent to the volume of the case sample $\left(500 \mathrm{TCID}_{50}\right)$; no RdRp expression was detected. The relative expression of RdRp was calculated by using rfhSP-D untreated cells (0 $\mu \mathrm{M}$ rfhSP-D) infected with respective samples as the calibrator. Data are provided in Table E1, and data for representative cases $(n=2)$ are presented as the mean of triplicates $(n=3)$. Error bars represent \pm SEM. Significance (compared with control sample [cells + virus]) was determined using the two-way ANOVA test. ${ }^{* * *} P<0.0001$.

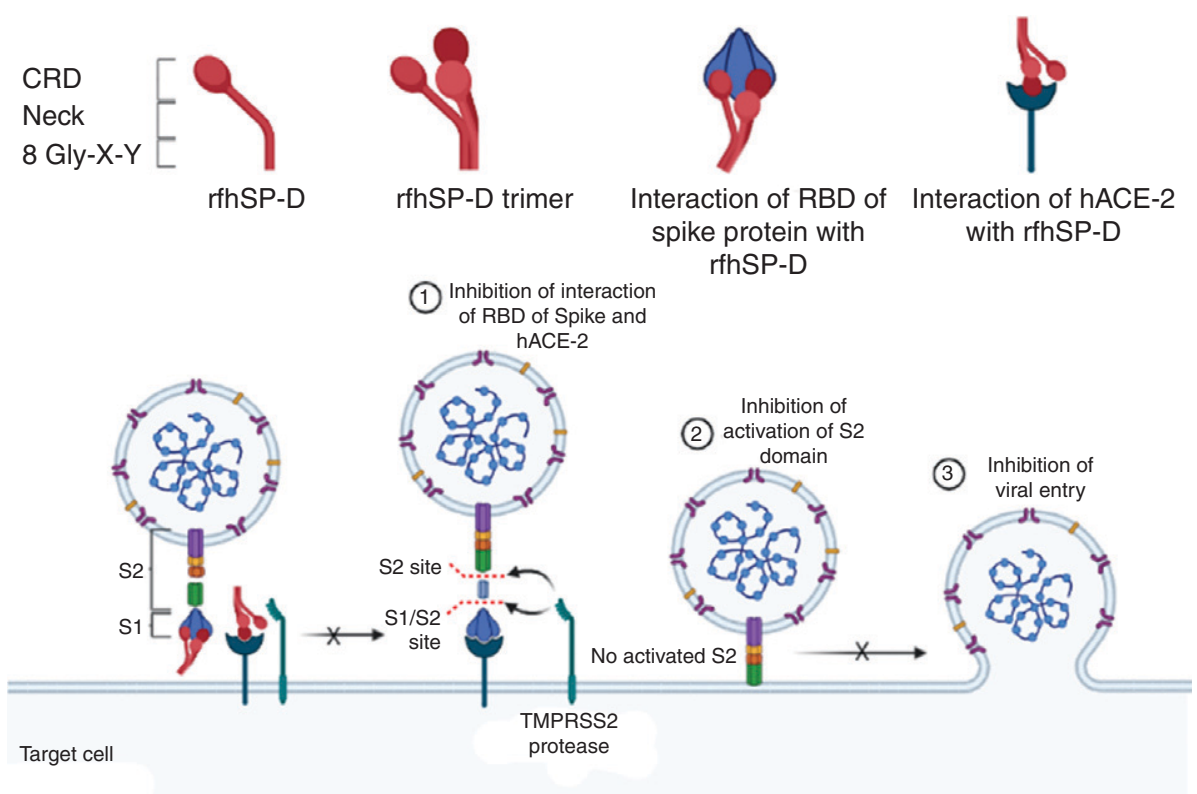

Figure 9. rfhSP-D binds spike/RBD and hACE-2 and inhibits SARS-CoV-2 infection and replication. Interaction of rfhSP-D with RBD/spike of SARS-CoV-2 and hACE-2 inhibit the interaction of RBD/spike and hACE-2, which is essential for viral entry into the host cells. This dual interaction of rfhSP-D plausibly causes the significant inhibition of infection and replication of SARS-CoV-2 in clinical samples. The figure was created using BioRender.com. 
adenosine analog, functions by incorporating itself into nascent viral RNA chains, which results in premature termination, thereby effectively inhibiting viral RNA synthesis (31). Downregulated RdRp expression in the remdesivir-treated samples clearly validated the platform for evaluating viral replication using clinical samples. A dose-dependent reduction of the RdRp mRNA expression in Vero cells challenged with rfhSP-

D-pretreated SARS-CoV-2-positive clinical samples at a higher fold-change than remdesivir suggested a highly potent anti-SARS-CoV-2 activity of rfhSP-D.

Replication kinetic studies involving Vero cells infected with SARS-CoV-2 have demonstrated a significant synthesis of viral RNA at $\geqslant 6$ hours after infection (32). As such, any viral RNA detected 1 to 2 hours after infection could be considered to have come from the infecting viral particles and not from subsequent viral RNA synthesis or viral replication. Hence, to confirm whether rfhSP-D played a role in inhibiting SARSCoV-2 infection, Vero cells were infected with SARS-CoV-2 clinical samples at a high concentration (500 $\mathrm{TCID}_{50}$; MOI, 0.05) for 2 hours. In accordance with the previous reports, lack of any significant effect of remdesivir on the Ct values of Vero cells, challenged with clinical samples, validated the assay format (33). Reduced RdRp transcripts in presence of rfhSP-D demonstrated the ability of rfhSP-D to act as an entry inhibitor against SARS-CoV-2. These results suggest that rfhSP-D is a potential candidate to be used as an $\mathrm{S}$ protein-based inhibitor against SARS-CoV-2 infection. With its established safety in vivo and therapeutic efficacy against several respiratory pathogens, rfhSP-D will effectively combat the nosocomial coinfections in patients with COVID-19.

There exists a dysregulated proinflammatory cytokine response without protective IFNs in response to SARS-CoV2-mediated lung tissue damage, leading to acute respiratory distress syndrome. The concentrations of SP-D were significantly altered in the BAL of patients with acute respiratory distress syndrome and were strong predictors of poor prognosis $(34,35)$. Persistent complement activation leads to microangiopathy, leading to hypoxia in vital organs. The current therapeutic strategy comprises an antiviral such as remdesivir and immunosuppressants such as corticosteroids (which can potentially invite opportunistic infections). Importantly, there is a need to rapidly clear cell debris or damage-associated molecular patterns and polarize the immune response toward a protective one and regulate the complement activation. The rfhSP-D is capable of dampening the "cytokine storm" by rapid clearance of the virus-infected cells and strengthening the lung capacity by restoring homeostasis (36).

rfhSP-D has been previously shown to inhibit human immunodeficiency virus 1 entry as well as successfully thwart the cytokine storm in an ex vivo model of human vaginal tissue (37). SP-D has a compelling role in correcting lung pathophysiology and injury (38). It is likely that SP-D functions as an opsonin after binding to the $S$ protein and helps in viral clearance. As a complementand antibody-independent neutralizing agent against SARS-CoV-2, rfhSP-D may be a viable alternative as an inhalation formulation to control COVID-19 infection in immunocompromised/immunodeficient people and in individuals who can not be be vaccinated due to related health complications. Our promising results warrant further studies in COVID-19 animal models, such as mice humanized with human ACE-2 and Syrian hamsters (Mesocricetus auratus), to better understand the impact of rfhSP-D in the microenvironment of the respiratory system (39).

Author disclosures are available with the text of this article at www.atsjournals.org.

Ethics Statement: The project was approved by the institutional ethics committee of Institute of Liver and Biliary Sciences, Delhi, (IEC/2020/ $80 / M A 04)$ on July 20,2020 . The committee waived off the written informed consent in due consideration of the request, as these samples were stored in the facility; anonymized aliquots of the samples were provided for the study.

Acknowledgment: The authors thank Dr. Smita Mahale (Director, ICMR-NIRRH) and Dr Shiv K. Sarin (Director, ILBS) for their support. The study was reviewed and recommended by ICMR Expert Review Committee. The authors also thank Dr. Nivedita Gupta and Dr. Raman Gangakhedkar of ICMR-ECD for their support. The study was reviewed by BIRAC-PACE expert panel.

\section{References}

1. Gorbalenya AE, Baker SC, Baric RS, de Groot RJ, Drosten C, Gulyaeva AA, et al.; Coronaviridae Study Group of the International Committee on Taxonomy of $\mathrm{V}$. The species Severe acute respiratory syndrome-related coronavirus: classifying 2019-nCoV and naming it SARS-CoV-2. Nat Microbiol 2020;5:536-544.

2. Zhu N, Zhang D, Wang W, Li X, Yang B, Song J, et al.; China Novel Coronavirus Investigating and Research Team. A novel coronavirus from patients with pneumonia in China, 2019. N Engl $\mathrm{J}$ Med 2020;382:727-733.

3. World Health Organization. COVID-19 weekly epidemiological update 24 November 2020, Geneva, Switzerland: World Health Organization; 2020. Available from: https://www.who.int/publications/m/item/weeklyepidemiological-update-24-november-2020.

4. Baughn LB, Sharma N, Elhaik E, Sekulic A, Bryce AH, Fonseca R. Targeting TMPRSS2 in SARS-CoV-2 infection. Mayo Clin Proc 2020;95:1989-1999.

5. National Institutes of Health. Therapeutic management of adults with COVID-19. Bethesda, MD: National Institutes of Health; 2021 [accessed 2021 Feb 2]. Available from: https://www.covid19treatmentguidelines. nih.gov/therapeutic-management/.
6. Carmo A, Pereira-Vaz J, Mota V, Mendes A, Morais C, da Silva AC, et al. Clearance and persistence of SARS-CoV-2 RNA in patients with COVID-19. J Med Virol 2020;92:2227-2231.

7. Al-Ahdal MN, Murugaiah V, Varghese PM, Abozaid SM, Saba I, AlQahtani AA, et al. Entry inhibition and modulation of pro-inflammatory immune response against influenza $A$ virus by a recombinant truncated surfactant protein D. Front Immunol 2018;9:1586.

8. Madsen J, Gaiha GD, Palaniyar N, Dong T, Mitchell DA, Clark HW. Surfactant Protein D modulates HIV infection of both T-cells and dendritic cells. PLoS One 2013;8:e59047.

9. Kishore U, Bernal AL, Kamran MF, Saxena S, Singh M, Sarma PU, et al. Surfactant proteins SP-A and SP-D in human health and disease. Arch Immunol Ther Exp (Warsz) 2005;53:399-417.

10. Murugaiah V, Tsolaki AG, Kishore U. Collectins: innate immune pattern recognition molecules. Adv Exp Med Biol 2020;1204:75-127.

11. Wu YP, Liu ZH, Wei R, Pan SD, Mao NY, Chen B, et al. Elevated plasma surfactant protein $D(S P-D)$ levels and a direct correlation with antisevere acute respiratory syndrome coronavirus-specific $\lg G$ antibody in SARS patients. Scand J Immunol 2009;69:508-515.

12. Leth-Larsen R, Zhong F, Chow VTK, Holmskov U, Lu J. The SARS coronavirus spike glycoprotein is selectively recognized by lung surfactant protein D and activates macrophages. Immunobiology 2007;212:201-211. 
13. Funk CJ, Wang J, Ito Y, Travanty EA, Voelker DR, Holmes KV, et al. Infection of human alveolar macrophages by human coronavirus strain 229E. J Gen Virol 2012;93:494-503.

14. Binder M. TCID50 calculator. 2020 [accessed 2020]. Available from: https://www.klinikum.uni-heidelberg.de/fileadmin/inst_hygiene/ molekulare_virologie/Downloads/TCID50_calculator_v2_17-01-20_MB. xIsx.

15. Lan J, Ge J, Yu J, Shan S, Zhou H, Fan S, et al. Structure of the SARSCoV-2 spike receptor-binding domain bound to the ACE2 receptor. Nature 2020;581:215-220.

16. Xu C, Wang Y, Liu C, Zhang C, Han W, Hong X, et al. Conformational dynamics of SARS-CoV-2 trimeric spike glycoprotein in complex with receptor ACE2 revealed by cryo-EM. Sci Adv 2021;7:eabe5575.

17. Andrusier N, Nussinov R, Wolfson HJ. FireDock: fast interaction refinement in molecular docking. Proteins 2007:69:139-159.

18. Mashiach E, Schneidman-Duhovny D, Andrusier N, Nussinov R, Wolfson HJ. FireDock: a web server for fast interaction refinement in molecular docking. Nucleic Acids Res 2008;36:W229-W332.

19. Duhovny D, Nussinov R, Wolfson HJ. Efficient unbound docking of rigid molecules. Lect Notes Comput Sci 2002;2452:185-200.

20. Schneidman-Duhovny D, Inbar $Y$, Nussinov R, Wolfson HJ. PatchDock and SymmDock: servers for rigid and symmetric docking. Nucleic Acids Res 2005;33:W363-W367.

21. Rodrigues CHM, Myung Y, Pires DEV, Ascher DB. mCSM-PPI2: predicting the effects of mutations on protein-protein interactions. Nucleic Acids Res 2019;47:W338-W344.

22. Murugaiah V, Agostinis C, Varghese PM, Belmonte B, Vieni S, Alaql FA, et al. Hyaluronic acid present in the tumor microenvironment can negate the pro-apototic effect of a recombinant fragment of human surfactant protein D on breast cancer cells. Front Immunol 2020;11:1171.

23. Singh M, Madan T, Waters P, Parida SK, Sarma PU, Kishore U. Protective effects of a recombinant fragment of human surfactant protein $\mathrm{D}$ in a murine model of pulmonary hypersensitivity induced by dust mite allergens. Immunol Lett 2003;86:299-307.

24. Kaur A, Riaz MS, Murugaiah V, Varghese PM, Singh SK, Kishore U. A recombinant fragment of human surfactant protein $D$ induces apoptosis in pancreatic cancer cell lines via fas-mediated pathway. Front Immunol 2018;9:1126.

25. Kumar J, Murugaiah V, Sotiriadis G, Kaur A, Jeyaneethi J, Sturniolo I, et al. Surfactant protein $\mathrm{D}$ as a potential biomarker and therapeutic target in ovarian cancer. Front Oncol 2019;9:542.

26. Thakur G, Prakash G, Murthy V, Sable N, Menon S, Alrokayan SH, et al. Human SP-D acts as an innate immune surveillance molecule against androgen-responsive and androgen-resistant prostate cancer cells. Front Oncol 2019;9:565.
27. Benton DJ, Wrobel AG, Xu P, Roustan C, Martin SR, Rosenthal PB, et al. Receptor binding and priming of the spike protein of SARS-CoV-2 for membrane fusion. Nature 2020;588:327-330.

28. Shang J, Ye G, Shi K, Wan Y, Luo C, Aihara H, et al. Structural basis of receptor recognition by SARS-CoV-2. Nature 2020;581:221-224.

29. Lui I, Zhou XX, Lim SA, Elledge SK, Solomon P, Rettko NJ, et al. Trimeric SARS-CoV-2 Spike interacts with dimeric ACE2 with limited intra-Spike avidity [preprint]. bioRxiv; 2020. Available from: https://www. biorxiv.org/content/10.1101/2020.05.21.109157v1.

30. Pandit H, Gopal S, Sonawani A, Yadav AK, Qaseem AS, Warke H, et al. Surfactant protein $D$ inhibits HIV-1 infection of target cells via interference with gp120-CD4 interaction and modulates proinflammatory cytokine production. PLoS One 2014;9:e102395.

31. Eastman RT, Roth JS, Brimacombe KR, Simeonov A, Shen M, Patnaik $S$, et al. Remdesivir: a Review of its discovery and development leading to emergency use authorization for treatment of COVID-19. ACS Cent Sci 2020;6:672-683.

32. Ogando NS, Dalebout TJ, Zevenhoven-Dobbe JC, Limpens RWAL, van der Meer Y, Caly L, et al. SARS-coronavirus-2 replication in Vero E6 cells: replication kinetics, rapid adaptation and cytopathology. J Gen Virol 2020;101:925-940.

33. Wang M, Cao R, Zhang L, Yang X, Liu J, Xu M, et al. Remdesivir and chloroquine effectively inhibit the recently emerged novel coronavirus (2019-nCoV) in vitro. Cell Res 2020;30:269-271.

34. Greene KE, Wright JR, Steinberg KP, Ruzinski JT, Caldwell E, Wong WB, et al. Serial changes in surfactant-associated proteins in lung and serum before and after onset of ARDS. Am J Respir Crit Care Med 1999;160:1843-1850.

35. Park J, Pabon M, Choi AMK, Siempos II, Fredenburgh LE, Baron RM, et al. Plasma surfactant protein-D as a diagnostic biomarker for acute respiratory distress syndrome: validation in US and Korean cohorts. BMC Pulm Med 2017;17:204.

36. Vandivier RW, Ogden CA, Fadok VA, Hoffmann PR, Brown KK, Botto M, et al. Role of surfactant proteins A, D, and C1q in the clearance of apoptotic cells in vivo and in vitro: calreticulin and CD91 as a common collectin receptor complex. J Immunol 2002;169:3978-3986.

37. Pandit H, Kale K, Yamamoto H, Thakur G, Rokade S, Chakraborty P, et al. Surfactant protein $D$ reverses the gene signature of transepithelial HIV-1 passage and restricts the viral transfer across the vaginal barrier. Front Immunol 2019;10:264.

38. Knudsen L, Ochs M, Mackay R, Townsend P, Deb R, Mühlfeld C, et al. Truncated recombinant human SP-D attenuates emphysema and type II cell changes in SP-D deficient mice. Respir Res 2007;8:70.

39. Muñoz-Fontela C, Dowling WE, Funnell SGP, Gsell PS, Riveros-Balta AX, Albrecht RA, et al. Animal models for COVID-19. Nature 2020;586:509-515. 